\title{
A realistic intersecting D6-brane model after the first LHC run
}

Tianjun Li, ${ }^{a, b}$ D.V. Nanopoulos, ${ }^{c, d, e}$ Shabbar Raza ${ }^{a}$ and Xiao-Chuan Wang ${ }^{a}$

${ }^{a}$ State Key Laboratory of Theoretical Physics and

Kavli Institute for Theoretical Physics China (KITPC), Institute of Theoretical Physics, Chinese Academy of Sciences, Beijing 100190, P.R. China

${ }^{b}$ School of Physical Electronics, University of Electronic Science and Technology of China, Chengdu 610054, P.R. China

${ }^{c}$ George P. and Cynthia W. Mitchell Institute for Fundamental Physics, Texas A\&M University, College Station, Texas 7r843, U.S.A.

${ }^{d}$ Astroparticle Physics Group, Houston Advanced Research Center (HARC), Mitchell Campus, Woodlands, Texas 77381, U.S.A.

${ }^{e}$ Academy of Athens, Division of Natural Sciences, 28 Panepistimiou Avenue, Athens 10679, Greece

E-mail: tli@itp.ac.cn, dimitri@physics.tamu.edu, shabbar@itp.ac.cn, xcwang@itp.ac.cn 
ABSTRACT: With the Higgs boson mass around $125 \mathrm{GeV}$ and the LHC supersymmetry search constraints, we revisit a three-family Pati-Salam model from intersecting D6-branes in Type IIA string theory on the $\mathbf{T}^{\mathbf{6}} /\left(\mathbb{Z}_{\mathbf{2}} \times \mathbb{Z}_{\mathbf{2}}\right)$ orientifold which has a realistic phenomenology. We systematically scan the parameter space for $\mu<0$ and $\mu>0$, and find that the gravitino mass is generically heavier than about $2 \mathrm{TeV}$ for both cases due to the Higgs mass low bound $123 \mathrm{GeV}$. In particular, we identify a region of parameter space with the electroweak fine-tuning as small as $\Delta_{E W} \sim 24-32(3-4 \%)$. In the viable parameter space which is consistent with all the current constraints, the mass ranges for gluino, the first two-generation squarks and sleptons are respectively $[3,18] \mathrm{TeV},[3,16] \mathrm{TeV}$, and $[2,7] \mathrm{TeV}$. For the third-generation sfermions, the light stop satisfying $5 \sigma$ WMAP bounds via neutralino-stop coannihilation has mass from 0.5 to $1.2 \mathrm{TeV}$, and the light stau can be as light as $800 \mathrm{GeV}$. We also show various coannihilation and resonance scenarios through which the observed dark matter relic density is achieved. Interestingly, the certain portions of parameter space has excellent $t-b-\tau$ and $b-\tau$ Yukawa coupling unification. Three regions of parameter space are highlighted as well where the dominant component of the lightest neutralino is a bino, wino or higgsino. We discuss various scenarios in which such solutions may avoid recent astrophysical bounds in case if they satisfy or above observed relic density bounds. Prospects of finding higgsino-like neutralino in direct and indirect searches are also studied. And we display six tables of benchmark points depicting various interesting features of our model. Note that the lightest neutralino can be heavy up to $2.8 \mathrm{TeV}$, and there exists a natural region of parameter space from low-energy fine-tuning definition with heavy gluino and first two-generation squarks/sleptons, we point out that the $33 \mathrm{TeV}$ and $100 \mathrm{TeV}$ proton-proton colliders are indeed needed to probe our D-brane model.

KEYwords: Supersymmetry Phenomenology, Strings and branes phenomenology

ARXIV EPRINT: 1406.5574 


\section{Contents}

1 Introduction 1

2 Phenomenological constraints and scanning procedure $\quad 3$

3 Fine-tuning $\quad \mathbf{5}$

3.1 Electroweak scale fine-tuning 6

$\begin{array}{ll}3.2 \text { High scale fine-tuning } & 6\end{array}$

$\begin{array}{lll}4 & \text { Numerical results } & 7\end{array}$

$\begin{array}{llr}5 & \text { Discussions and conclusion } & 29\end{array}$

\section{Introduction}

String theory is one of the most promising candidates for quantum gravity. Thus, the string phenomenology goal is to construct the Standard Model (SM) or Supersymmetric SMs (SSMs) from string theory with moduli stabilization and without chiral exotics, and try to make unique predictions which can probed at the Large Hadron Collider (LHC) and other future experiments. It is well-known that four kinds of string models have been studied extensively: (1) The heterotic $E_{8} \times E_{8}$ string model building. The SSMs can be constructed via the orbifold compactifications ${ }^{1}[3-5]$ and the Calabi-Yau manifold compactifications [6]. ${ }^{2}$ (2) The free fermionic string model building. The realistic models with clean particle spectra such as the standard-like models, Pati-Salam models, and flipped SU(5) models have been constructed at the Kac-Moody level one [9-15]. ${ }^{3}$ (3) The D-brane model building. Two kinds of such models have been studied: (i) Intersecting D-brane models [17$28]^{4}$ and [30-32]; (ii) Orientifolds of Gepner models [33].5 (4) The F-theory model building for the $\mathrm{SU}(5)$, flipped $\mathrm{SU}(5)$, and $\mathrm{SU}(3)_{C} \times \mathrm{SU}(2)_{L} \times \mathrm{SU}(2)_{R} \times \mathrm{U}(1)_{B-L}$ models [35-43].

For the intersecting D-brane model building, the realistic SM fermion Yukawa couplings can be realized only in the Pati-Salam models. The three-family Pati-Salam models have been constructed systematically in Type IIA string theory on the $\mathbf{T}^{\mathbf{6}} /\left(\mathbb{Z}_{\mathbf{2}} \times \mathbb{Z}_{\mathbf{2}}\right)$ orientifold with intersecting D6-branes [23], and two of us (TL and DVN) with Chen and Mayes found that one model has a realistic phenomenology: the tree-level gauge coupling unification is realized naturally at the string scale, the Pati-Salam gauge symmetry can be broken to the SM close to the string scale, the small number of extra chiral exotic states may be decoupled

\footnotetext{
${ }^{1}$ See $[1,2]$ and references therein.

${ }^{2}$ See also $[7,8]$ and references therein.

${ }^{3}$ See also [16] and references therein.

${ }^{4}$ See also [29] and references therein.

${ }^{5}$ See also [34] and references therein.
} 
via the Higgs mechanism and strong dynamics, the SM fermion masses and mixings can be explained, the low-energy supersymmetric particle spectra might potentially be tested at the LHC, and the observed dark matter relic density may be generated for the lightest neutralino as the lightest supersymmetric particle (LSP), etc [44, 45]. As far as we know, this is indeed one of the best globally consistent string models.

On the other hand, for the first run of the LHC, the big success is obviously the discovery of a SM-like Higgs boson with mass $m_{h}$ around $125 \mathrm{GeV}$ in July 2012 [46, 47], which is a little bit too large for the Minimal SSM (MSSM). Such large Higgs boson mass in the MSSM requires the multi-TeV top squarks with small mixing or TeV-scale top squarks with large mixing. In addition, the LHC supersymmetry (SUSY) searches have given strong constraints on the pre-LHC viable parameter space. For instance, the gluino mass $m_{\tilde{g}}$ should be heavier than about $1.7 \mathrm{TeV}$ if the first two-generation squark mass $m_{\tilde{q}}$ is around the gluino mass $m_{\tilde{q}} \sim m_{\tilde{g}}$, and heavier than about $1.3 \mathrm{TeV}$ for $m_{\tilde{q}} \gg m_{\tilde{g}}[48,49]$.

Therefore, we should update the phenomenological study of this intersecting D-brane model. For this purpose, we have systematically scan the viable parameter space by considering $\mu<0$ and $\mu>0$ scenarios where $\mu$ is the bilinear Higgs mass term. We show that there indeed exists such viable parameter space which satisfies the collider and astrophysical bounds including the Higgs boson mass in the range $[123,127] \mathrm{GeV}$. In particular, the absolute value of $\mu$ can be as small as $300 \mathrm{GeV}$ in a region of parameter space, where the electroweak fine-tuning (EWFT) is small around $\Delta_{E W} \sim 24-32(3-4 \%)$. We identify another region of parameter space with $|\mu| \lesssim 500 \mathrm{GeV}$ and $\Delta_{E W} \lesssim 300$, where gluino masses are from 3 to $7 \mathrm{TeV}$, and the first two-generation squarks and sleptons are in the mass ranges of $[4,7] \mathrm{TeV}$ and $[2,4] \mathrm{TeV}$, respectively. Because such parameter space is natural from the low-energy fine-tuning definition while the gluino and first two-generation squarks/sleptons are out of the reach of $14 \mathrm{TeV}$ LHC, this will provide a strong motivation for the $33 \mathrm{TeV}$ and $100 \mathrm{TeV}$ proton-proton colliders. There is some visible preference to achieve the viable parameter space consistent with constraints for $\mu<0$ case, but this is just an artifact of lack of statistics for $\mu>0$. Moreover, in order to have the Higgs boson mass from $123 \mathrm{GeV}$ to $127 \mathrm{GeV}$, and satisfy the LHC low bounds on sparticles and the Bphysics bounds, we require gravitino mass $\gtrsim 2 \mathrm{TeV}$ for both cases of $\mu<0$ and $\mu>0$. We also present graphs in neutralino-sparticle planes showing various coannihilation scenarios such as neutralino-stau, neutralino-stop, neutralino-gluino, and $A$-resonance solutions. The solutions, which are consistent with the observed relic density, have gluino masses from 3 to $18 \mathrm{TeV}$. We also note that in our present data consistent with all bounds, the first two generation squarks are in the mass range $[3,16] \mathrm{TeV}$ and the first two generation sleptons can be heavier than $2 \mathrm{TeV}$ but less than $6 \mathrm{TeV}$. On the other hand for third family squarks, the NLSP light stop satisfying $5 \sigma$ WMAP bounds is in the mass of $0.5-1.2 \mathrm{TeV}$, in case of third family slepton, the light stau can be as light as $800 \mathrm{GeV}$. We have checked status of $t-b-\tau$ and $b-\tau$ Yukawa unification (YU) scenarios with both signs of $\mu$ in our data. For $\mu<0$ we find solutions with $10 \%$ or better YU with typical heavy spectra. The best YU we have achieved in our data set is about $5 \%$ consistent with all the constraints including the observed dark matter relic density bound. On the other hand, we do not have better than $12 \%$ YU $t-b-\tau$ for $\mu>0$ case. Since we did not perform any dedicate searches to study YU 
in this project otherwise we may have solutions with much better YU. Relaxing the $t-b-\tau$ YU constraint to $b-\tau$ YU, we have plenty of solutions with $100 \%$ YU. For the points with $\Omega h^{2} \gtrsim 1$ where the lightest neutralino is almost a pure bino, we introduce a lighter state axino $\tilde{a}$ as the LSP. Thus, the lightest neutralino is the Next to the LSP (NLSP) and can decay to axino via $\tilde{\chi}_{1}^{0} \rightarrow \gamma \tilde{a}$. We calculate the lifetime of the NLSP neutralino for various choices of the axion decay constant $f_{a}$ in our data. For $f_{a}>10^{14} \mathrm{GeV}$, the lifetime of the NLSP bino is more than 1 second and may be ruled out by Big Bang Nucleosynthesis $(\mathrm{BBN})$ constraints. We also note that in our data, there are solutions where the lightest neutralino can be a bino, wino, or higgsino type. The lightest neutralino masses are more than $1 \mathrm{TeV}$ for both cases $(\mu<0$ and $\mu>0)$ in the wino-type solutions, while they are less than $1 \mathrm{TeV}$ in the bino-type solutions and in the mass range of $150-600 \mathrm{GeV}$ in the higgsino-type solutions. Recent studies showed that the scenario with pure wino as dark matter is under siege $[50,51]$. In our model, the relic density of the wino dominant lightest neutralino can be smaller than the correct relic density, and then the above constraint can be escaped. Otherwise, to solve this problem, we suggest that the wino dominant neutralino is the NLSP and may decay to $\tilde{a} \gamma$ and hence fulfil the relic density bounds, or we may invoke R-parity violation. Similarly, the higgsino-type solutions suffer underabundance of relic density problem. In such a case we assume that the higgsino-type neutralino makes up only a fraction of the dark matter relic density and the remaining abundance is comprised of axions. We also display graphs for direct and indirect searches for dark matter for our higgsino-like solutions and show that these solutions will be observed or ruled out by the XENON1T experiment. Finally, we present six tables of benchmark points, three for each sign of $\mu$. These points depict various interesting scenarios of our model, namely points with minimum EWFT, various coannihilation and resonance solutions, bino-type, wino-type and higgsino-type solutions. Furthermore, because the lightest neutralino can be heavier than $1 \mathrm{TeV}$ and up to about $2.8 \mathrm{TeV}$, how to search for such scenario at the $14 \mathrm{TeV}$ LHC is still a challenging question. In short, we do need the $33 \mathrm{TeV}$ and $100 \mathrm{TeV}$ proton-proton colliders to probe such D-brane model.

This paper is organized as follows. In section 2 we outline details of the supersymmetry breaking (SSB) parameters, the range of values employed in our scan, the scanning procedure and the relevant experimental constraints that we have employed. In section 3 we briefly describe our definition of EWFT and High scale (GUT) fine-tuning. We discuss results of our scans in section 4. A summary and conclusions are given in section 5 .

\section{Phenomenological constraints and scanning procedure}

In our realistic intersecting D-brane model, if we do not consider $\mathrm{CP}$ violation, the supersymmetry breaking (SSB) soft terms from the non-zero F-terms $F^{u^{i}}$ and $F^{s}$ can be parametrized by $\Theta_{1}, \Theta_{2}, \Theta_{3}, \Theta_{4} \equiv \Theta_{s}$, and gravitino mass $m_{3 / 2}$ where $\sum_{i=1}^{4} \Theta_{i}^{2}=1$ [45]. Thus, we can reparametrize $\Theta_{i}$ with $i=1,2,3$ in terms of $\gamma_{1}, \gamma_{2}$ and $\Theta_{4}$ as follows

$$
\begin{aligned}
\alpha & \equiv 2 \pi \gamma_{1}, \\
\beta & \equiv 2 \pi \gamma_{2},
\end{aligned}
$$




$$
\begin{aligned}
& \Theta_{1}=\cos (\beta) \cos (\alpha) \sqrt{1-\Theta_{4}^{2}}, \\
& \Theta_{2}=\cos (\beta) \sin (\alpha) \sqrt{1-\Theta_{4}^{2}}, \\
& \Theta_{3}=\sin (\beta) \sqrt{1-\Theta_{4}^{2}} .
\end{aligned}
$$

Thus, the supersymmetry breaking soft terms are [45]

$$
\begin{aligned}
M_{1} & =\left(0.519 \Theta_{1}+0.346 \Theta_{2}+0.866 \Theta_{3}\right) m_{3 / 2} \\
M_{2} & =\left(0.866 \Theta_{2}-0.866 \Theta_{4}\right) m_{3 / 2}, \\
M_{3} & =\left(0.866 \Theta_{2}+0.866 \Theta_{3}\right) m_{3 / 2} \\
A_{0} & =\left(-1.111 \Theta_{1}-0.621 \Theta_{2}+0.245 \Theta_{3}-0.245 \Theta_{4}\right) m_{3 / 2}, \\
m_{L} & =\sqrt{\left(1.0+0.899 \Theta_{1}^{2}-0.518 \Theta_{2}^{2}-0.849 \Theta_{3}^{2}-1.418 \Theta_{4}^{2}-0.557 \Theta_{1} \Theta_{2}-0.557 \Theta_{3} \Theta_{4}\right)} m_{3 / 2}, \\
m_{R} & =\sqrt{\left(1.0-1.418 \Theta_{1}^{2}-0.849 \Theta_{2}^{2}-0.518 \Theta_{3}^{2}+0.899 \Theta_{4}^{2}-0.557 \Theta_{1} \Theta_{2}-0.557 \Theta_{3} \Theta_{4}\right)} m_{3 / 2}, \\
m_{H_{u}} & =m_{H_{d}}=\sqrt{\left(1.0-\left(1.5 \Theta_{3}^{2}\right)-\left(1.5 \Theta_{4}^{2}\right)\right)} m_{3 / 2},
\end{aligned}
$$

where $M_{1,2,3}$ are the gauginos masses respectively for $\mathrm{U}(1)_{Y}, \mathrm{SU}(2)_{L}$ and $\mathrm{SU}(3)_{c}$ gauge groups, $A_{0}$ is the trilinear scalar coupling, $m_{L}$ and $m_{R}$ are the soft mass terms respectively for the left-handed and right-handed squarks and sleptons, and $m_{H_{u, d}}$ are the SSB Higgs soft mass terms.

We employ the ISAJET 7.84 package [52] to perform random scans over the parameter space given below. In this package, the weak scale values of gauge and third generation Yukawa couplings are evolved to $M_{\mathrm{GUT}}$ via the MSSM renormalization group equations (RGEs) in the $\overline{D R}$ regularization scheme. We do not strictly enforce the unification condition $g_{3}=g_{1}=g_{2}$ at $M_{\mathrm{GUT}}$, since a few percent deviation from unification can be assigned to unknown GUT-scale threshold corrections [53-55]. With the boundary conditions given at $M_{\mathrm{GUT}}$, all the SSB parameters, along with the gauge and Yukawa couplings, are evolved back to the weak scale $M_{\mathrm{Z}}$.

In evaluating Yukawa couplings the SUSY threshold corrections [56] are taken into account at the common scale $M_{\mathrm{SUSY}}=\sqrt{m_{\tilde{t}_{L}} m_{\tilde{t}_{R}}}$. The entire parameter set is iteratively run between $M_{\mathrm{Z}}$ and $M_{\mathrm{GUT}}$ using the full 2-loop RGEs until a stable solution is obtained. To better account for leading-log corrections, one-loop step-beta functions are adopted for gauge and Yukawa couplings, and the SSB parameters $m_{i}$ are extracted from RGEs at appropriate scales $m_{i}=m_{i}\left(m_{i}\right)$. The RGE-improved 1-loop effective potential is minimized at an optimized scale $M_{\text {SUSY }}$, which effectively accounts for the leading 2-loop corrections. Full 1-loop radiative corrections are incorporated for all sparticle masses.

The requirement of radiative electroweak symmetry breaking (REWSB) [57-61] puts an important theoretical constraint on the parameter space. Another important constraint comes from limits on the cosmological abundance of stable charged particle [62]. This excludes regions in the parameter space where charged SUSY particles, such as $\tilde{\tau}_{1}$ or $\tilde{t}_{1}$, become the LSP. We accept only those solutions for which one of the neutralinos is the LSP and saturates the dark matter relic abundance bound observed by WMAP9. 
We have performed Markov-chain Monte Carlo (MCMC) scans for the following parameter range

$$
\begin{aligned}
& 0 \leq \gamma_{1} \leq 1, \\
& 0 \leq \gamma_{2} \leq 1, \\
& 0 \leq \Theta_{4} \leq 1, \\
& 1 \leq m_{3 / 2} \leq 10 \mathrm{TeV}, \\
& 2 \leq \tan \beta \leq 60,
\end{aligned}
$$

where $\tan \beta$ is the ratio of the vacuum expectation values (VEVs) of two Higgs fields. We use $m_{t}=173.3 \mathrm{GeV}$ [63] , and $m_{b}^{\overline{D R}}\left(M_{\mathrm{Z}}\right)=2.83 \mathrm{GeV}$ which is hard-coded into ISAJET. We have done our scans with both $\mu<0$ and $\mu>0$, and find that our results are not too sensitive to one or two sigma variation in the value of $m_{t}$ [64].

In scanning the parameter space, we employ the Metropolis-Hastings algorithm as described in $[65,66]$. The collected data points all satisfy the requirement of REWSB, with the neutralino in each case being the LSP. After collecting the data, we require the following bounds (inspired by the LEP2 experiment) on particle masses:

$$
\begin{aligned}
m_{\tilde{t}_{1}}, m_{\tilde{b}_{1}} & \gtrsim 100 \mathrm{GeV}, \\
m_{\tilde{\tau}_{1}} & \gtrsim 105 \mathrm{GeV}, \\
m_{\tilde{\chi}_{1}^{ \pm}} & \gtrsim 103 \mathrm{GeV} .
\end{aligned}
$$

We also use IsaTools package [67-71] and ref. [72] to implement the following B-physics constraints:

$$
\begin{aligned}
0.8 \times 10^{-9} \leq \mathrm{BR}\left(B_{s} \rightarrow \mu^{+} \mu^{-}\right) \leq 6.2 \times 10^{-9}(2 \sigma) & {[73], } \\
2.99 \times 10^{-4} \leq \mathrm{BR}(b \rightarrow s \gamma) \leq 3.87 \times 10^{-4}(2 \sigma) & {[74], } \\
0.15 \leq \frac{\mathrm{BR}\left(B_{u} \rightarrow \tau \nu_{\tau}\right)_{\mathrm{MSSM}}}{\operatorname{BR}\left(B_{u} \rightarrow \tau \nu_{\tau}\right)_{\mathrm{SM}}} \leq 2.41(3 \sigma) & {[75] }
\end{aligned}
$$

In addition to above constraints, we impose the following bounds from the LHC and WMAP9

$$
\begin{aligned}
& m_{h}=123-127 \mathrm{GeV} \quad[76-78], \\
& m_{\tilde{g}} \gtrsim 1.7 \mathrm{TeV} \text { (for } m_{\tilde{g}} \sim m_{\tilde{q}} \text { ) }[48,49] \text {, } \\
& m_{\tilde{g}} \gtrsim 1.3 \mathrm{TeV} \text { (for } m_{\tilde{g}} \ll m_{\tilde{q}} \text { ) }[48,49], \\
& 0.0913 \leq \Omega_{\mathrm{CDM}} h^{2}(\mathrm{WMAP} 9) \leq 0.1363(5 \sigma) \quad \text { [79] }
\end{aligned}
$$

As far as the muon anomalous magnetic moment $a_{\mu}$ is concerned, we require that the benchmark points are at least as consistent with the data as the Standard Model.

\section{Fine-tuning}

We use the latest (7.84) version of ISAJET [52] to calculate the fine-tuning (FT) conditions at the electroweak scale (EW) $M_{E W}$ and at the high scale $\left(M_{H S}\right)$. Brief description of these parameters is given in this section. 
The $\mathrm{Z}$ boson mass $M_{Z}$, after including the one-loop effective potential contributions to the tree level MSSM Higgs potential, is given by the following relation:

$$
\frac{M_{Z}^{2}}{2}=\frac{\left(m_{H_{d}}^{2}+\Sigma_{d}^{d}\right)-\left(m_{H_{u}}^{2}+\Sigma_{u}^{u}\right) \tan ^{2} \beta}{\tan ^{2} \beta-1}-\mu^{2},
$$

where $\Sigma_{u}^{u}$ and $\Sigma_{d}^{d}$ are the contributions coming from the one-loop effective potential defined in [80]) and $\tan \beta \equiv\left\langle H_{u}\right\rangle /\left\langle H_{d}\right\rangle$. All parameters in eq. (3.1) are defined at the $M_{E W}$.

\subsection{Electroweak scale fine-tuning}

We follow [80] in order to measure the EW scale fine-tuning condition, the following definitions are used:

$$
C_{H_{d}} \equiv\left|m_{H_{d}}^{2} /\left(\tan ^{2} \beta-1\right)\right|, C_{H_{u}} \equiv\left|-m_{H_{u}}^{2} \tan ^{2} \beta /\left(\tan ^{2} \beta-1\right)\right|, C_{\mu} \equiv\left|-\mu^{2}\right|,
$$

with each $C_{\Sigma_{u, d}^{u, d}(k)}$ less than some characteristic value of order $M_{Z}^{2}$. Here, $k$ labels the SM and SUSY particles that contribute to the one-loop Higgs potential. For the fine-tuning condition we have

$$
\Delta_{\mathrm{EW}} \equiv \max \left(C_{k}\right) /\left(M_{Z}^{2} / 2\right) .
$$

It is important to note that $\Delta_{E W}$ depends only on the weak scale parameters of the theory, therefore fixed by the particle spectrum. Hence, it is independent of how SUSY particle masses arise. Lower values of $\Delta_{E W}$ correspond to less fine tuning, for example, $\Delta_{E W}=10$ implies $\Delta_{E W}^{-1}=10 \%$ fine tuning. Moreover, this condition of EW scale fine-tuning is different from the fine-tuning definition in refs. [81, 82] beyond the tree level (for more details see [83]).

\subsection{High scale fine-tuning}

From eq. (3.1) it is evident that $\Delta_{E W}$ does not give any informations about the possible high scale origin of the parameters in the equation. In order to address fully the fine-tuning condition we need to write down weak-scale parameter $m_{H_{u, d}}^{2}$ in eq. (3.1) and with their explicit dependence on the (HS) as:

$$
m_{H_{u, d}}^{2}=m_{H_{u, d}}^{2}\left(M_{H S}\right)+\delta m_{H_{u, d}}^{2}, \quad \mu^{2}=\mu^{2}\left(M_{H S}\right)+\delta \mu^{2} .
$$

Here $m_{H_{u, d}}^{2}\left(M_{H S}\right)$ and $\mu^{2}\left(M_{H S}\right)$ are the corresponding parameters renormalized at the high scale, and $\delta m_{H_{u, d}}^{2}$, and $\delta \mu^{2}$ measure how the given parameter is changed due to Renormalization Group Equation (RGE) evolution. Eq. (3.1) can be re-expressed in the form

$$
\begin{aligned}
\frac{m_{Z}^{2}}{2}= & \frac{\left(m_{H_{d}}^{2}\left(M_{H S}\right)+\delta m_{H_{d}}^{2}+\Sigma_{d}^{d}\right)-\left(m_{H_{u}}^{2}\left(M_{H S}\right)+\delta m_{H_{u}}^{2}+\Sigma_{u}^{u}\right) \tan ^{2} \beta}{\tan ^{2} \beta-1} \\
& -\left(\mu^{2}\left(M_{H S}\right)+\delta \mu^{2}\right) .
\end{aligned}
$$

As we did before, we follow ref. [80] and introduce the following parameters

$$
B_{H_{d}} \equiv\left|m_{H_{d}}^{2}\left(M_{H S}\right) /\left(\tan ^{2} \beta-1\right)\right|, \quad \quad B_{\delta H_{d}} \equiv\left|\delta m_{H_{d}}^{2} /\left(\tan ^{2} \beta-1\right)\right|,
$$




$$
\begin{aligned}
B_{H_{u}} & \equiv\left|-m_{H_{u}}^{2}\left(M_{H S}\right) \tan ^{2} \beta /\left(\tan ^{2} \beta-1\right)\right|, & B_{\mu} & \equiv\left|\mu^{2}\left(M_{H S}\right)\right|, \\
B_{\delta H_{u}} & \equiv\left|-\delta m_{H_{u}}^{2} \tan ^{2} \beta /\left(\tan ^{2} \beta-1\right)\right|, & B_{\delta \mu} & \equiv\left|\delta \mu^{2}\right|,
\end{aligned}
$$

and the high scale fine-tuning measure $\Delta_{\mathrm{HS}}$ is defined to be

$$
\Delta_{\mathrm{HS}} \equiv \max \left(B_{i}\right) /\left(M_{Z}^{2} / 2\right) .
$$

In short, $\Delta_{E W}$ includes information about the minimal amount of fine-tuning present in the low scale model for a given SUSY spectrum, while $\Delta_{H S}$ better represents the finetuning that is present in high scale model.

\section{Numerical results}

In figure 1, we present graphs for various parameter given in eq. (2.3). The left and the right panels show solutions for $\mu<0$ and $\mu>0$ scenarios, respectively. Color coding is given as, grey points satisfy REWSB and neutralino as an LSP conditions. Aqua points satisfy the mass bounds and B-physics bounds. Magenta points are subset of aqua points and also represent $123 \mathrm{GeV} \leqslant m_{h} \leqslant 127 \mathrm{GeV}$. Red points are subset of magenta points and also satisfy WMAP9 $5 \sigma$ bounds.

We see that in our scans, in $\Theta_{1}-\Theta_{2}$ plane for both cases, the range of red points for $\Theta_{1}$ is $-0.6 \lesssim \Theta_{1} \lesssim 0.6$, but most of the points are concentrated in the range -0.4 to 0.4, while for $\Theta_{2}$ most of the points are in the range of large values 0.4-0.8. But we also have some red points -0.6 to -0.4 . On the other hand, magenta points can be more or less anywhere in the plot. We see that for $\Theta_{1}$, we have solutions for its entire range in contrast to $\Theta_{2}$ where points mostly have relatively large absolute values. In $\Theta_{1}-\Theta_{3}$ plane we see that red points favor positive values of $\Theta_{1}$ and $\Theta_{3}$ as we have also seen in $\Theta_{1}-\Theta_{2}$ plane. We also see some red points for small negative values of $\Theta_{1}$ and but large negative values of $\Theta_{3}$. Magenta points are every where but in contrast to $\Theta_{1}-\Theta_{2}$ plane, here large density of points are around the centre of the plot. In the last panel we have plot in $\Theta_{3}-\Theta_{2}$ plane. Here too, we see that the red points lie mostly in large positive ranges of $\Theta_{2}$ and $\Theta_{3}$. In case of magenta points, as compared to other panels, here we have some kind of polarisation and we do not have magenta points in the center.

We calculate (SSB) parameters using eqs. (2.3) and (2.2). We present our results in figure 2. Color coding and panel description are same as in figure 1. In the top left and right panels we present plots in $M_{1}-M_{2}$ plane. We note that there are some minor differences. In left panel we see that there is a patch of red points around $M_{1} \sim-1 \mathrm{TeV}$ and $M_{2} \sim 4 \mathrm{TeV}$ as compare to right panel where we have some red points around $M_{1} \sim$ $-5 \mathrm{TeV}$ and $M_{2} \sim 2 \mathrm{TeV}$. In case of magenta points, there are points up to $M_{2} \sim-12 \mathrm{TeV}$ in the left panel as compared to right panel. Similarly, we also note minor differences in other panels of the figure. In figure 3 , plots in $m_{L}-m_{R}, \tan \beta-m_{H_{u, d}}$ and $\tan \beta-A_{0}$ planes are displayed. Color coding is same as in figure 1. The left panels represent $\mu<0$ and the right panels represent $\mu>0$ cases. In $m_{L}-m_{R}$ plane we see that the left and right panels have almost similar data spread with some minor differences. For example, in the left panel we have more points around $m_{R} \sim 12 \mathrm{TeV}$, while in the right panel the 

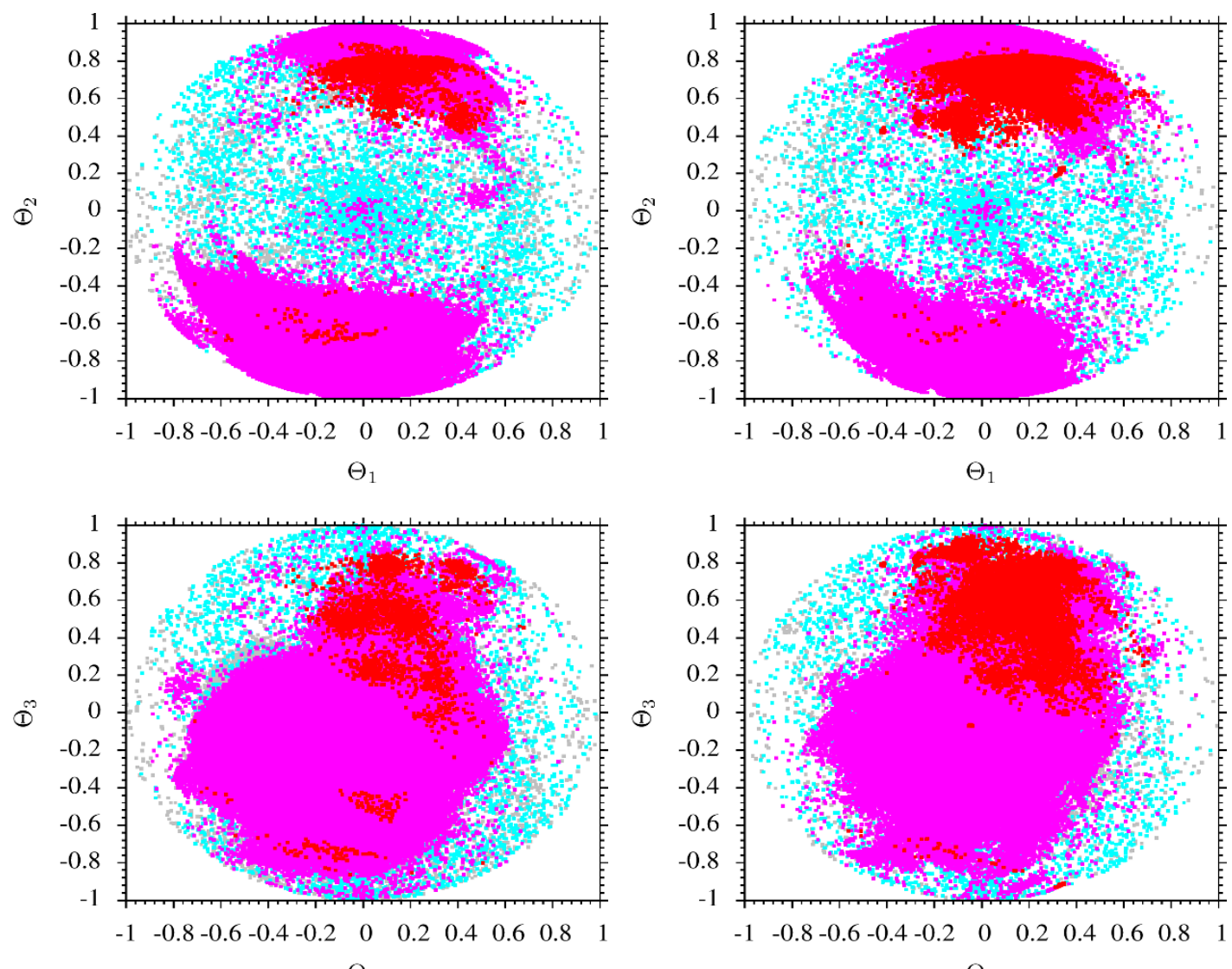

$\Theta_{1}$
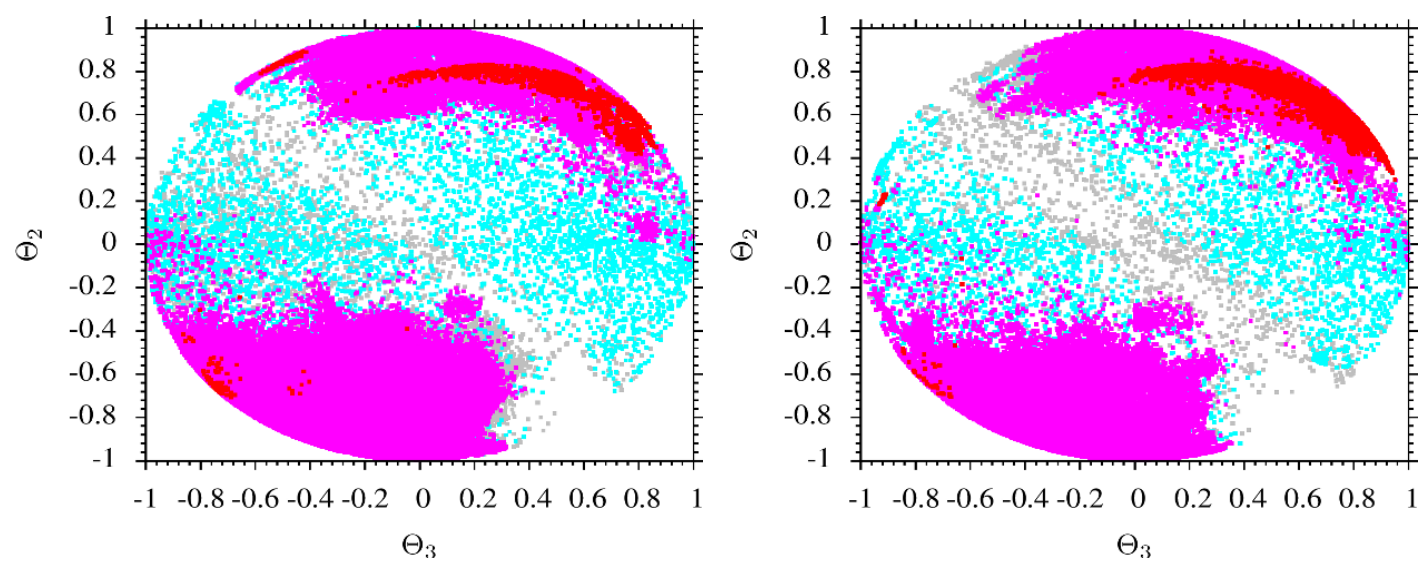

Figure 1. Plots in $\Theta_{1}-\Theta_{2}, \Theta_{1}-\Theta_{3}$ and $\Theta_{3}-\Theta_{2}$ planes for $\mu<0$ (left panels) and $\mu>0$ (right panels). Grey points satisfy the REWSB and yield LSP neutralino. Aqua points satisfy all the mass bounds and B-physics bounds. Magenta points are subset of aqua points and also represent $123 \mathrm{GeV} \leqslant m_{h} \leqslant 127 \mathrm{GeV}$. Red points are subset of magenta points and also satisfy WMAP9 $5 \sigma$ bounds. 

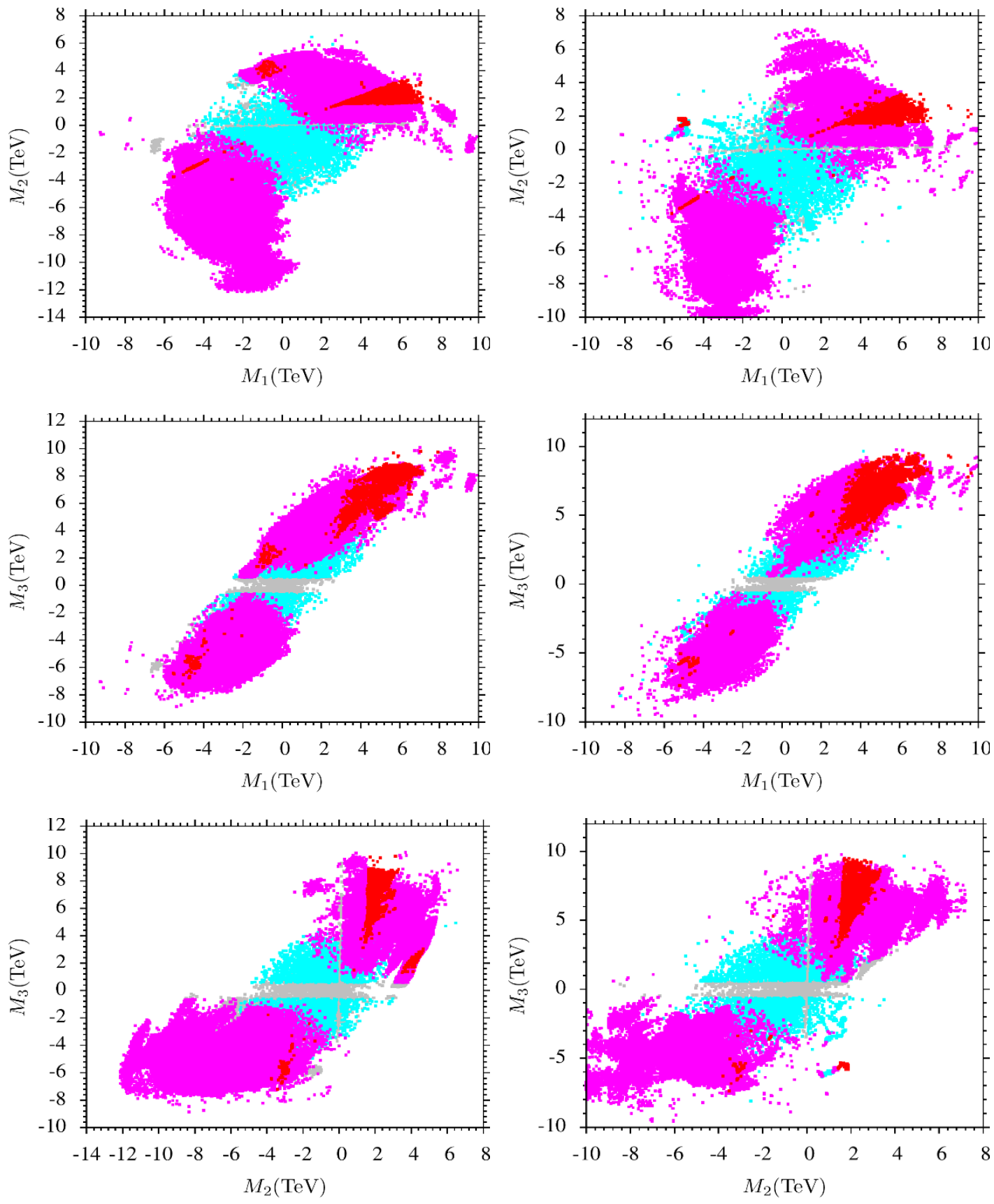

Figure 2. Plots in $M_{1}-M_{2}, M_{1}-M_{3}$ and $M_{3}-M_{2}$ planes. Color coding and panel description are same as in figure 1. 

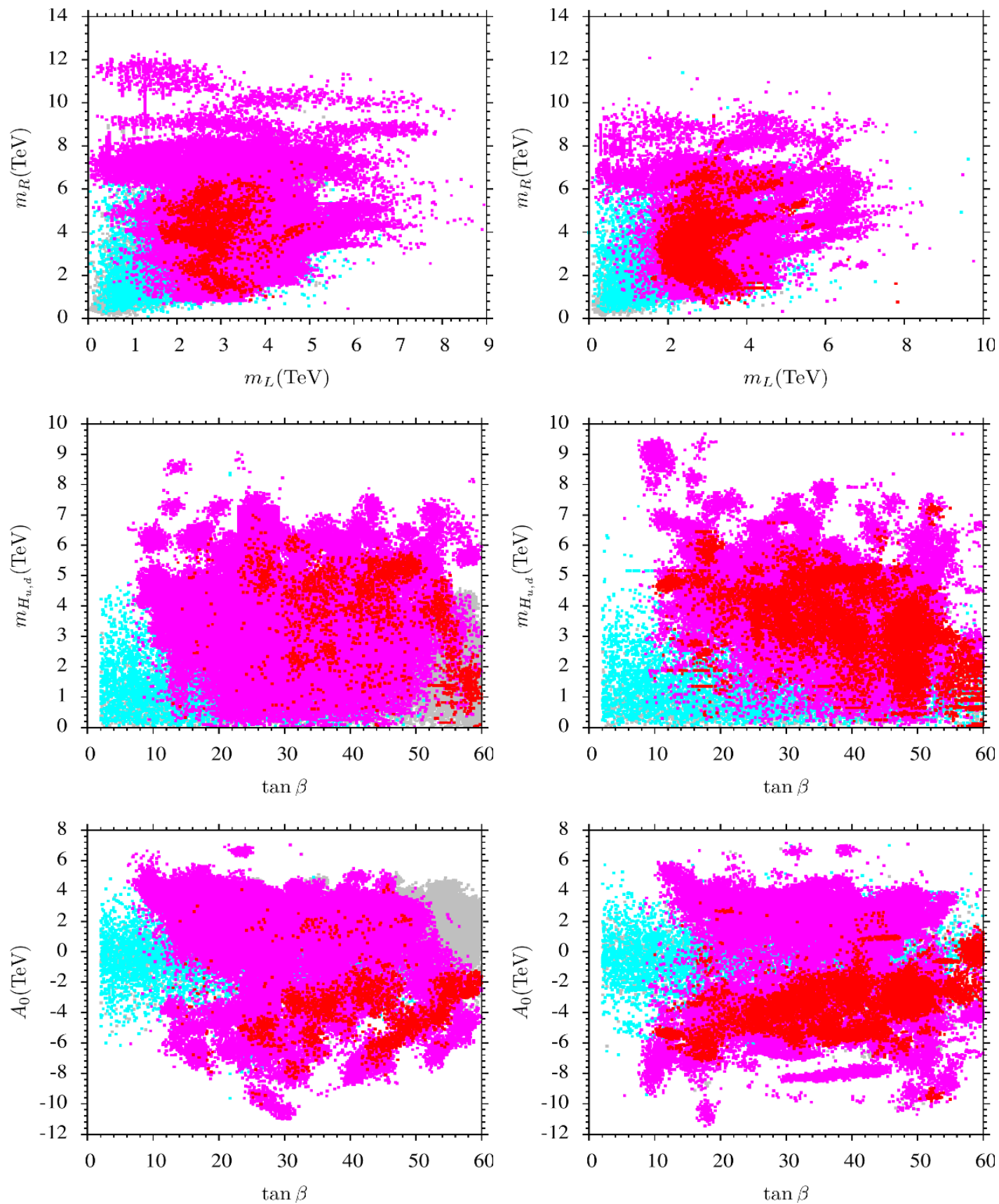

Figure 3. Plots in $m_{L}-m_{R}, \tan \beta-m_{H_{u, d}}$ and $\tan \beta-A_{0}$ planes. Color coding and panel description are same as in figure 1. 

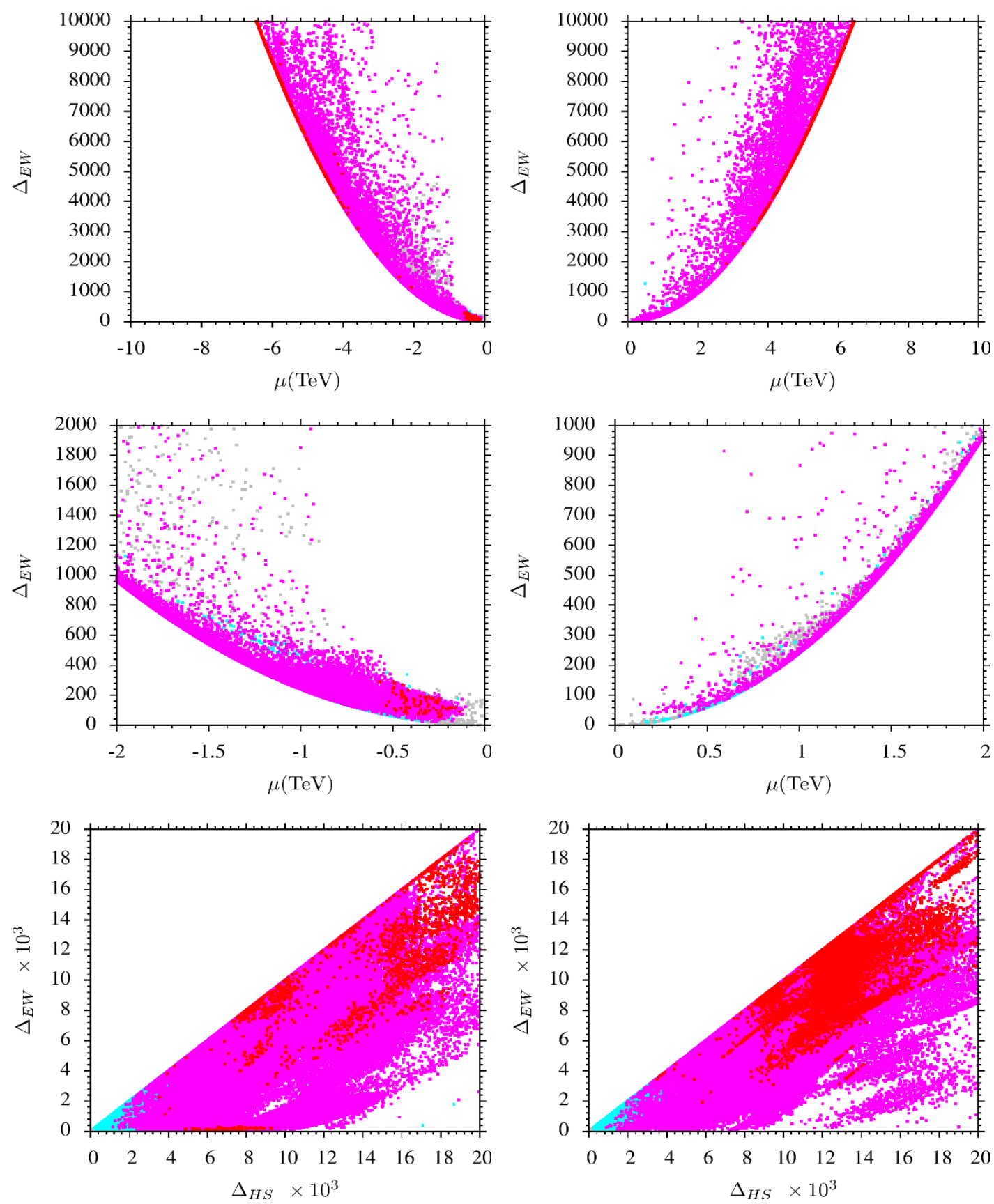

Figure 4. Plots in $\mu-\Delta_{E W}$ and $\Delta_{H S}-\Delta_{E W}$ planes. Color coding and panel description are same as in figure 1. 

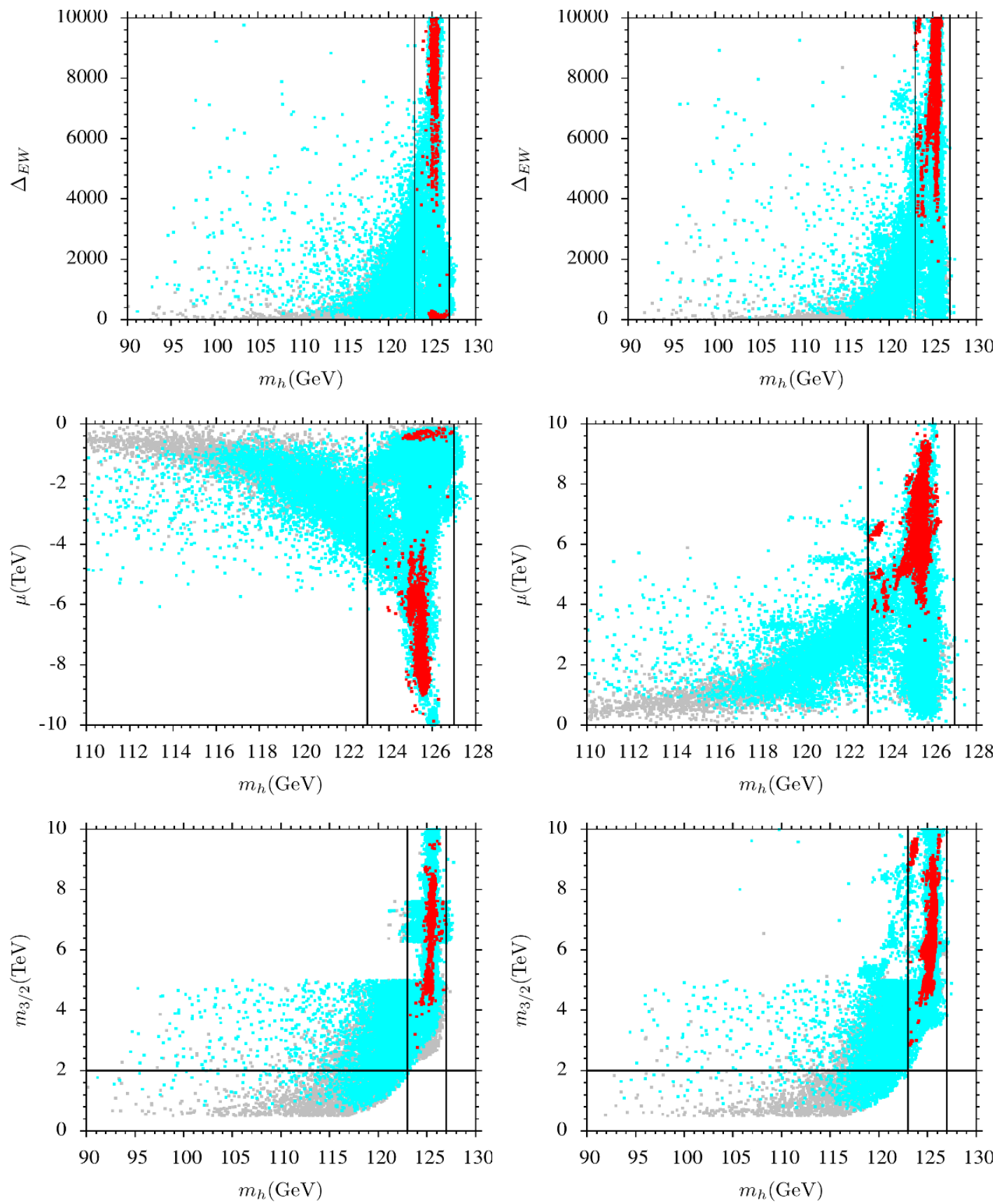

Figure 5. Plots in $m_{h}-\Delta_{E W}, m_{h}-\mu$ and $m_{h}-m_{3 / 2}$ planes. Grey points satisfy REWSB and yield LSP neutralino. Aqua points satisfy all the mass bounds and B-physics bounds. Red points are subset of aqua points and also satisfy the WMAP9 $5 \sigma$ bounds. 


\begin{tabular}{|c|ccccc|}
\hline & Point 1 & Point 2 & Point 3 & Point 4 & Point 5 \\
\hline$m_{L}$ & 2763.6 & 4077.8 & 1353.6 & 3125.3 & 2599.1 \\
$m_{R}$ & 2187.1 & 3343.4 & 1401.1 & 1414.8 & 4991.8 \\
$M_{1}$ & -379.47 & -441.48 & -865.62 & 4894.5 & 5792.8 \\
$M_{2}$ & 2850.2 & 3957.4 & -2681.8 & 1802.4 & 2082.9 \\
$M_{3}$ & 1495.1 & 2206.2 & -1194.1 & 4953.4 & 8736.4 \\
$A_{0}$ & -3144.2 & -5006.1 & 2127.6 & -2884.4 & -2626.3 \\
$\tan \beta$ & 12.8 & 23 & 11.1 & 57.7 & 46.8 \\
$m_{H_{u}}=m_{H_{d}}$ & 3470 & 5127.6 & 1857 & 1778.4 & 3692.7 \\
\hline$\mu$ & -290 & -244 & -1110 & -5500 & -8688 \\
$\Delta_{E W}$ & 24 & 56 & 297 & 7279 & 18163 \\
$\Delta_{H S}$ & 2941 & 6395 & 1125 & 7663 & 20667 \\
\hline$m_{h}$ & 124 & 125 & 123 & 125 & 126 \\
$m_{H}$ & 3828 & 5146 & 2712 & 1369 & 6761 \\
$m_{A}$ & 3803 & 5112 & 2695 & 1360 & 6717 \\
$m_{H^{ \pm}}$ & 3828 & 5146 & 2713 & 1373 & 6762 \\
\hline$m_{\tilde{\chi}_{1,2}^{0}}$ & 177,300 & 202,255 & 377,1120 & 1503,2221 & 1697,2647 \\
$m_{\tilde{\chi}_{3,4}^{0}}$ & 310,2375 & 273,3314 & 1122,2209 & 5403,5403 & 8561,8561 \\
$m_{\tilde{\chi}_{1,2}^{ \pm}}$ & 285,2348 & 238,3274 & 1139,2193 & 1506,5356 & 1700,8478 \\
\hline$m_{\tilde{g}}$ & 3281 & 4725 & 2630 & 9818 & 16760 \\
\hline$m_{\tilde{u}_{L, R}}$ & 4239,3490 & 6112,5133 & 3069,2626 & 8934,8510 & 14308,15024 \\
$m_{\tilde{t}_{1,2}}$ & 1240,3536 & 1933,4958 & 1273,2620 & 6955,7659 & 12604,12906 \\
\hline$m_{\tilde{d}_{L, R}}$ & 4240,3491 & 6112,5136 & 3070,2622 & 8934,8456 & 14308,14994 \\
$m_{\tilde{b}_{1,2}}$ & 3386,3579 & 4751,5025 & 2565,2638 & 7066,7617 & 12672,13902 \\
\hline$m_{\tilde{\nu}_{1,2}}$ & 3294 & 4767 & 2175 & 3415 & 3015 \\
$m_{\tilde{\nu}_{3}}$ & 3268 & 4624 & 2162 & 3221 & 2551 \\
\hline$m_{\tilde{e}_{L, R}}$ & 3291,2187 & 4763,3342 & 2176,1435 & 3423,2273 & 3006,5410 \\
$m_{\tilde{\tau}_{1,2}}$ & 2094,3264 & 2898,4620 & 1386,2161 & 1511,3218 & 2527,4810 \\
\hline$\sigma_{S I}(\mathrm{pb})$ & $2.41 \times 10^{-9}$ & $8.34 \times 10^{-9}$ & $5.97 \times 10^{-11}$ & $2.78 \times 10^{-12}$ & $5.52 \times 10^{-13}$ \\
$\sigma_{S D}(\mathrm{pb})$ & $3.39 \times 10^{-5}$ & $1.29 \times 10^{-4}$ & $8.55 \times 10^{-8}$ & $1.52 \times 10^{-9}$ & $1.71 \times 10^{-10}$ \\
$\Omega_{C D M} h^{2}$ & 0.731 & 0.101 & 21.3 & 0.126 & 0.115 \\
$R_{t b \tau}$ & 7.44 & 4.04 & 8.68 & 1.05 & 1.85 \\
$R_{b \tau}$ & 1.35 & 1.35 & 1.36 & 1.04 & 1.35 \\
\hline & & & & & \\
\hline
\end{tabular}

Table 1. All the masses are in units of $\mathrm{GeV}$ and $\mu<0$. All points satisfy the sparticle mass bounds, and B-physics constraints described in section 2. Points 1 and 2 represent the minimal value of $\Delta_{E W}$ not consistent and consistent with the WMAP9 $5 \sigma$ bounds, while points 3-5 correspond respectively to the minimal value of $\Delta_{H S}$, best points with $t-b-\tau$ and $b-\tau$ YU, an example of heavy gluino solution. Points 3 and 4 also satisfy the WMAP9 $5 \sigma$ bounds. 


\begin{tabular}{|c|cccc|}
\hline & Point 1 & Point 2 & Point 3 & Point 4 \\
\hline$m_{L}$ & 2608.7 & 4050.1 & 3521.9 & 6589.5 \\
$m_{R}$ & 2270.8 & 2651.8 & 3022.8 & 6501 \\
$M_{1}$ & -4372.3 & -2542.1 & -4090.1 & -2646.7 \\
$M_{2}$ & -2924.2 & -3947.6 & -2720.5 & -6538.3 \\
$M_{3}$ & -5426.5 & -1767.6 & -4397.9 & -487.58 \\
$A_{0}$ & 2123.4 & 4103.5 & 4070.6 & 6423.1 \\
$\tan \beta$ & 33.6 & 23.5 & 45.8 & 32.6 \\
$m_{H_{u}}=m_{H_{d}}$ & 2216.6 & 3258.7 & 3615 & 4428.1 \\
\hline$\mu$ & -5691 & -2418 & -4801 & -3572 \\
$\Delta_{E W}$ & 7793 & 1489 & 5546 & 3070 \\
$\Delta_{H S}$ & 8745 & 4049 & 8489 & 7761 \\
\hline$m_{h}$ & 126 & 127 & 126 & 125 \\
$m_{H}$ & 5307 & 4246 & 3827 & 5267 \\
$m_{A}$ & 5272 & 4218 & 3802 & 5233 \\
$m_{H}$ & 5308 & 4247 & 3828 & 5267 \\
\hline$m_{\tilde{\chi}_{1,2}^{0}}$ & 1958,2437 & 1155,2425 & 1863,2285 & 1237,3591 \\
$m_{\tilde{\chi}_{3,4}^{0}}$ & 5718,5720 & 2429,3327 & 4815,4817 & 3592,5542 \\
$m_{\tilde{\chi}_{1,2}^{ \pm}}$ & 2444,5719 & 2467,3303 & 2289,4817 & 3646,5501 \\
\hline$m_{\tilde{g}}$ & 10673 & 3847 & 8807 & 1308 \\
\hline$m_{\tilde{u}_{L, R}}$ & 9526,9362 & 5644,4153 & 8369,8082 & 7717,6555 \\
$m_{\tilde{t}_{1,2}}$ & 7694,8601 & 1186,4747 & 6173,7097 & 3084,6172 \\
\hline$m_{\tilde{d}_{L, R}}$ & 9527,9324 & 5645,4120 & 8369,8046 & 7717,6533 \\
$m_{\tilde{b}_{1,2}}$ & 8562,8820 & 3751,4817 & 7022,7081 & 5649,6210 \\
\hline$m_{\tilde{\nu}_{1,2}}$ & 3242 & 4760 & 3958 & 7749 \\
$m_{\tilde{\nu}_{3}}$ & 3081 & 4648 & 3505 & 7370 \\
\hline$m_{\tilde{e}_{L, R}}$ & 3256,3089 & 4754,2801 & 3964,3367 & 7742,6566 \\
$m_{\tilde{\tau}_{1,2}}$ & 2356,3089 & 2360,4640 & 2097,3504 & 5591,7355 \\
\hline$\sigma_{S I}(\mathrm{pb})$ & $2.30 \times 10^{-12}$ & $1.85 \times 10^{-11}$ & $3.70 \times 10^{-12}$ & $3.63 \times 10^{-12}$ \\
$\sigma_{S D}(\mathrm{pb})$ & $1.11 \times 10^{-10}$ & $3.88 \times 10^{-9}$ & $2.51 \times 10^{-10}$ & $6.93 \times 10^{-10}$ \\
$\Omega_{C D M} h^{2}$ & 0.114 & 0.114 & 0.121 & 0.055 \\
$R_{t b \tau}$ & 2.5 & 3.9 & 1.73 & 2.5 \\
$R_{b \tau}$ & 1.37 & 1.38 & 1.44 & 1.34 \\
\hline & & & & \\
\hline & & & & \\
\hline
\end{tabular}

Table 2. All the masses are in units of $\mathrm{GeV}$ and $\mu<0$. All points satisfy the sparticle mass bounds, and B-physics constraints described in section 2. Points 1, 2, 3 and 4 display neutralinostau, neutralino-stop, $A$-resonance, and neutralino-gluino coannihilation respectively. Point 4 is the case where relic density is below the WMAP9 $5 \sigma$ bounds. 


\begin{tabular}{|c|cccc|}
\hline & Point 1 & Point 2 & Point 3 & Point 4 \\
\hline$m_{L}$ & 4342.3 & 2443.1 & 3047.1 & 2537.4 \\
$m_{R}$ & 3727.6 & 1830.1 & 1485.7 & 2570.6 \\
$M_{1}$ & -657.78 & -886.71 & 5041.4 & -4748.2 \\
$M_{2}$ & 4058.9 & -4758.7 & 1623.6 & -2511.2 \\
$M_{3}$ & 2107.5 & -2435 & 5143.7 & -5673.4 \\
$A_{0}$ & -5395.7 & 3635.3 & -2951.9 & 1813.3 \\
$\tan \beta$ & 26 & 18.1 & 57.2 & 31.9 \\
$m_{H_{u}}=m_{H_{d}}$ & 5307.9 & 3954.6 & 1689.5 & 1112.0 \\
\hline$\mu$ & -333 & -312 & -5696 & -6129 \\
$\Delta_{E W}$ & 79 & 38 & 7807 & 9039 \\
$\Delta_{H S}$ & 6869 & 3813 & 8100 & 9082 \\
\hline$m_{h}$ & 126 & 126 & 125 & 125 \\
$m_{H}$ & 5140 & 4682 & 1834 & 5509 \\
$m_{A}$ & 5106 & 4651 & 1822 & 5473 \\
$m_{H^{ \pm}}$ & 5141 & 4682 & 1836 & 5509 \\
\hline$m_{\tilde{\chi}_{1,2}^{0}}$ & 298,345 & 307,323 & 1347,2287 & 2084,2157 \\
$m_{\tilde{\chi}_{3,4}^{0}}$ & 366,3406 & 399,3946 & 5596,5596 & 6157,6158 \\
$m_{\tilde{\chi}_{1,2}^{ \pm}}$ & 325,3367 & 332,3920 & 1350,5548 & 2091,6158 \\
\hline$m_{\tilde{g}}$ & 4549 & 5075 & 10172 & 11140 \\
\hline$m_{\tilde{u}_{L, R}}$ & 6208,5266 & 5705,4643 & 9177,8827 & 9826,9820 \\
$m_{\tilde{t}_{1,2}}$ & 1924,4943 & 2038,4824 & 7257,7896 & 8226,8947 \\
\hline$m_{\tilde{d}_{L, R}}$ & 6208,5268 & 5706,4644 & 9177,8767 & 9827,9784 \\
$m_{\tilde{b}_{1,2}}$ & 4775,5014 & 4419,4880 & 7413,7856 & 8907,9337 \\
\hline$m_{\tilde{\nu}_{1,2}}$ & 5027 & 3850 & 3313 & 3063 \\
$m_{\tilde{\nu}_{3}}$ & 4833 & 3783 & 3118 & 2936 \\
\hline$m_{\tilde{e}_{L, R}}$ & 5022,3731 & 3849,1849 & 3322,2360 & 3077,3093 \\
$m_{\tilde{\tau}_{1,2}}$ & 3161,4828 & 1537,3781 & 1637,3112 & 2793,2961 \\
\hline$\sigma_{S I}(\mathrm{pb})$ & $9.66 \times 10^{-9}$ & $7.41 \times 10^{-9}$ & $1.02 \times 10^{-12}$ & $2.55 \times 10^{-11}$ \\
$\sigma_{S D}(\mathrm{pb})$ & $7.14 \times 10^{-5}$ & $4.72 \times 10^{-5}$ & $1.25 \times 10^{-9}$ & $1.45 \times 10^{-9}$ \\
$\Omega_{C D M} h^{2}$ & 0.119 & 0.03 & 0.091 & $0.14 \times 10^{-5}$ \\
$R_{t b \tau}$ & 3.55 & 5.2 & 1.10 & 2.0 \\
$R_{b \tau}$ & 1.37 & 1.34 & 1.10 & 1.37 \\
\hline & & & & \\
\hline$x_{1}$ & & & & \\
\hline
\end{tabular}

Table 3. All the masses are in this table are in units of $\mathrm{GeV}$ and $\mu<0$. All this points satisfy the sparticle mass and B-physics constraints described in section 2. Point 1 represents bino-like neutralino, point 2 displays higgsino like neutralino, point 3 and point 4 are examples of wino-like neutralino. Point 2 and point 4 do not satisfy WMAP9 $5 \sigma$ bounds. 


\begin{tabular}{|c|cccc|}
\hline & Point 1 & Point 2 & Point 3 & Point 4 \\
\hline$m_{L}$ & 1992.4 & 1541.5 & 3525.1 & 3632.8 \\
$m_{R}$ & 1598.5 & 1659.5 & 3466.1 & 5259.2 \\
$M_{1}$ & -507.03 & -911.52 & -3825.5 & 6933.9 \\
$M_{2}$ & -3790.9 & -2613.2 & -2531.4 & 1916.5 \\
$M_{3}$ & -1951.9 & -922.33 & -2993.5 & 9495 \\
$A_{0}$ & 2679.3 & 2189.1 & 1957.5 & 525.13 \\
$\tan \beta$ & 18 & 22.4 & 31.7 & 40.2 \\
$m_{H_{u}}=m_{H_{d}}$ & 3199.1 & 1676.6 & 662.98 & 4072.4 \\
\hline$\mu$ & 350 & 1083 & 4293 & 8968 \\
$\Delta_{E W}$ & 32 & 286 & 4435 & 19351 \\
$\Delta_{H S}$ & 2502 & 964 & 4438 & 22605 \\
\hline$m_{h}$ & 125 & 123 & 124 & 126 \\
$m_{H}$ & 3789 & 2344 & 3602 & 8695 \\
$m_{A}$ & 3765 & 2328 & 3578 & 8638 \\
$m_{H^{ \pm}}$ & 3790 & 2345 & 3603 & 8696 \\
\hline$m_{\tilde{\chi}_{1,2}^{0}}$ & 209,364 & 400,1092 & 1737,2124 & 1537,3176 \\
$m_{\tilde{\chi}_{3,4}^{0}}$ & 366,3133 & 1094,2158 & 4303,4304 & 8853,8853 \\
$m_{\tilde{\chi}_{1,2}^{ \pm}}$ & 342,3101 & 1067,2141 & 2130,4304 & 1541,8767 \\
\hline$m_{\tilde{g}}$ & 4131 & 2097 & 6216 & 18151 \\
\hline$m_{\tilde{u}_{L, R}}$ & 4631,3830 & 2818,2406 & 6451,6320 & 15622,16208 \\
$m_{\tilde{t}_{1,2}}$ & 1834,3936 & 1063,2321 & 5001,5687 & 13969,14327 \\
\hline$m_{\tilde{d}_{L, R}}$ & 4632,3833 & 2819,2402 & 6451,6272 & 15622,16155 \\
$m_{\tilde{b}_{1,2}}$ & 3663,3974 & 2230,2340 & 5646,5803 & 14293,15613 \\
\hline$m_{\tilde{\nu}_{1,2}}$ & 3104 & 2269 & 3915 & 3952 \\
$m_{\tilde{\nu}_{3}}$ & 3053 & 2215 & 3794 & 3566 \\
\hline$m_{\tilde{e}_{L, R}}$ & 3104,1603 & 2268,1692 & 3918,3734 & 3959,5821 \\
$m_{\tilde{\tau}_{1,2}}$ & 1391,3052 & 1528,2212 & 3467,3799 & 3549,5212 \\
\hline$\sigma_{S I}(\mathrm{pb})$ & $6.00 \times 10^{-10}$ & $1.07 \times 10^{-11}$ & $2.36 \times 10^{-12}$ & $5.58 \times 10^{-14}$ \\
$\sigma_{S D}(\mathrm{pb})$ & $1.75 \times 10^{-5}$ & $9.92 \times 10^{-8}$ & $3.35 \times 10^{-10}$ & $1.52 \times 10^{-10}$ \\
$\Omega_{C D M} h^{2}$ & 1.07 & 22.3 & 0.105 & 0.104 \\
\hline$R_{t b \tau}$ & 5.31 & 4.18 & 2.31 & 2.86 \\
$R_{b \tau}$ & 1.33 & 1.29 & 1.05 & 1.91 \\
\hline & & & & \\
\hline & & & & \\
\hline
\end{tabular}

Table 4. All the masses are in units of $\mathrm{GeV}$ and $\mu>0$. All points satisfy the sparticle mass bounds and B-physics constraints described in section 2. Points 1-4 respectively correspond to the minimal value of $\Delta_{E W}$, minimal value of $\Delta_{H S}$, best point with $b-\tau$ YU, and an example of heavy gluino solution. Points 3 and 4 also satisfy the WMAP9 $5 \sigma$ bounds. 


\begin{tabular}{|c|cccc|}
\hline & Point 1 & Point 2 & Point 3 & Point 4 \\
\hline$m_{L}$ & 2517.8 & 3734.8 & 2660.3 & 5549.1 \\
$m_{R}$ & 2187.1 & 1435 & 2837.6 & 7667.2 \\
$M_{1}$ & 1843.3 & 1871.4 & -4493 & -3325.8 \\
$M_{2}$ & 1796 & 1229.6 & -3017 & -4120.9 \\
$M_{3}$ & 3883 & 2215.4 & -5334.2 & -636.94 \\
$A_{0}$ & -971.61 & -5825.6 & 1714.9 & 2617.6 \\
$\tan \beta$ & 56.10 & 18.90 & 36.80 & 51.23 \\
$m_{H_{u}}=m_{H_{d}}$ & 2985.9 & 4082.3 & 1226.1 & 1762.9 \\
\hline$\mu$ & 3773 & 2579 & 5817 & 4968 \\
$\Delta_{E W}$ & 3425 & 1699 & 8142 & 5938 \\
$\Delta_{H S}$ & 5392 & 5721 & 8189 & 6542 \\
\hline$m_{h}$ & 124 & 127 & 125 & 123 \\
$m_{H}$ & 3296 & 4578 & 4047 & 2469 \\
$m_{A}$ & 3248 & 4548 & 4021 & 2453 \\
$m_{H}$ & 3271 & 4578 & 4048 & 2470 \\
\hline$m_{\tilde{\chi}_{1,2}^{0}}$ & 814,1488 & 833,1033 & 2046,2521 & 1542,3501 \\
$m_{\tilde{\chi}_{3,4}^{0}}$ & 3793,3793 & 2587,2588 & 5831,5832 & 4946,4948 \\
$m_{\tilde{\chi}_{1,2}^{ \pm}}$ & 1491,3793 & 1032,2612 & 2529,5831 & 3505,4939 \\
\hline$m_{\tilde{g}}$ & 7817 & 4691 & 10511 & 1662 \\
\hline$m_{\tilde{u}_{L, R}}$ & 7157,6999 & 5476,4206 & 9413,9390 & 6139,7788 \\
$m_{\tilde{t}_{1,2}}$ & 5672,6310 & 896,4523 & 7833,8321 & 4343,6129 \\
\hline$m_{\tilde{d}_{L, R}}$ & 7158,6994 & 5476,4190 & 9413,9349 & 6140,7765 \\
$m_{\tilde{b}_{1,2}}$ & 6264,6392 & 3906,4596 & 8272,8479 & 4417,6749 \\
\hline$m_{\tilde{\nu}_{1,2}}$ & 2753 & 3821 & 3327 & 6140 \\
$m_{\tilde{\nu}_{3}}$ & 2332 & 3721 & 3195 & 5532 \\
\hline$m_{\tilde{e}_{L, R}}$ & 2761,2284 & 3818,1569 & 3334,3272 & 6142,7759 \\
$m_{\tilde{\tau}_{1,2}}$ & 825,2333 & 1009,3724 & 2974,3213 & 5533,6765 \\
\hline$\sigma_{S I}(\mathrm{pb})$ & $7.49 \times 10^{-13}$ & $4.31 \times 10^{-12}$ & $1.12 \times 10^{-12}$ & $5.95 \times 10^{-13}$ \\
$\sigma_{S D}(\mathrm{pb})$ & $4.21 \times 10^{-10}$ & $1.77 \times 10^{-9}$ & $1.06 \times 10^{-10}$ & $1.68 \times 10^{-10}$ \\
$\Omega_{C D M} h^{2}$ & 0.137 & 0.296 & 0.126 & 0.178 \\
\hline$R_{t b \tau}$ & 2.13 & 4.96 & 1.83 & 1.48 \\
$R_{b \tau}$ & 2.12 & 1.40 & 1.08 & 1.21 \\
\hline & & & & \\
\hline & & & & \\
\hline
\end{tabular}

Table 5. All the masses are in units of $\mathrm{GeV}$ and $\mu>0$. All points satisfy the sparticle mass bounds, and B-physics constraints described in section 2. Points 1, 2, 3 and 4 display neutralinostau, neutralino-stop, $A$-resonance and neutralino-gluino coannihilation, respectively. Points 2 and 4 are the examples where relic density is little bit above the WMAP9 $5 \sigma$ bounds. 


\begin{tabular}{|c|cccc|}
\hline & Point 1 & Point 2 & Point 3 & Point 4 \\
\hline$m_{L}$ & 3620.5 & 2136.8 & 2537.4 & 3216.5 \\
$m_{R}$ & 1475.8 & 1248.8 & 2147.6 & 6057.0 \\
$M_{1}$ & 2080.6 & -713.72 & 5197.6 & -5042.8 \\
$M_{2}$ & 1109.9 & -3832.9 & 1992.9 & -2293.9 \\
$M_{3}$ & 2398.8 & -1991.3 & 6222.5 & -4933.4 \\
$A_{0}$ & -5687.8 & 3169.4 & -3322.2 & -1737.9 \\
$\tan \beta$ & 16 & 19 & 16.57 & 11.12 \\
$m_{H_{u}}=m_{H_{d}}$ & 4007.4 & 3358.8 & 2721.1 & 3843.9 \\
\hline$\mu$ & 2812 & 277 & 6507 & 5195 \\
$\Delta_{E W}$ & 1934 & 47 & 10189 & 6493 \\
$\Delta_{H S}$ & 5815 & 2770 & 11755 & 9839 \\
\hline$m_{h}$ & 126 & 126 & 125 & 123 \\
$m_{H}$ & 4752 & 3884 & 7054 & 6645 \\
$m_{A}$ & 4720 & 3859 & 7008 & 6602 \\
$m_{H^{ \pm}}$ & 4752 & 3885 & 7055 & 6646 \\
\hline$m_{\tilde{\chi}_{1,2}^{0}}$ & 926,930 & 267,289 & 1636,2359 & 1902,2295 \\
$m_{\tilde{\chi}_{3,4}^{0}}$ & 2819,2820 & 327,3178 & 6430,6430 & 5237,5238 \\
$m_{\tilde{\chi}_{1,2}^{ \pm}}$ & 927,2843 & 272,3149 & 1644,6373 & 1906,5233 \\
\hline$m_{\tilde{g}}$ & 5044 & 4199 & 12141 & 9899 \\
\hline$m_{\tilde{u}_{L, R}}$ & 5620,4515 & 4756,3755 & 10601,10561 & 8936,10358 \\
$m_{\tilde{t}_{1,2}}$ & 1805,4723 & 1394,4003 & 8684,9770 & 7989,8885 \\
\hline$m_{\tilde{d}_{L, R}}$ & 5621,4495 & 4756,3756 & 10602,10511 & 8937,10314 \\
$m_{\tilde{b}_{1,2}}$ & 4304,4780 & 3543,4055 & 9728,10370 & 8061,10228 \\
\hline$m_{\tilde{\nu}_{1,2}}$ & 3697 & 3218 & 2925 & 3610 \\
$m_{\tilde{\nu}_{3}}$ & 3626 & 3159 & 2864 & 3573 \\
\hline$m_{\tilde{e}_{L, R}}$ & 3696,1642 & 3217,1262 & 2950,2854 & 3599,6325 \\
$m_{\tilde{\tau}_{1,2}}$ & 1313,3628 & 897,3156 & 2729,2886 & 3580,6286 \\
\hline$\sigma_{S I}(\mathrm{pb})$ & $2.36 \times 10^{-11}$ & $9.03 \times 10^{-9}$ & $2.03 \times 10^{-12}$ & $8.48 \times 10^{-12}$ \\
$\sigma_{S D}(\mathrm{pb})$ & $1.16 \times 10^{-8}$ & $1.32 \times 10^{-4}$ & $6.81 \times 10^{-10}$ & $3.13 \times 10^{-9}$ \\
$\Omega_{C D M} h^{2}$ & 0.101 & 0.028 & 0.109 & $0.16 \times 10^{-5}$ \\
\hline$R_{t b \tau}$ & 6.05 & 4.98 & 6.25 & 8.2 \\
$R_{b \tau}$ & 1.41 & 1.33 & 1.51 & 1.25 \\
\hline & & & & \\
\hline & & & & \\
\hline
\end{tabular}

Table 6. All the masses are in this table are in units of $\mathrm{GeV}$ and $\mu>0$. All this points satisfy the sparticle mass and B-physics constraints described in section 2. Point 1 represents bino-like neutralino, point 2 displays higgsino like neutralino, point 3 and point 4 are examples of wino-like neutralino. Point 2 and point 4 do not satisfy WMAP9 $5 \sigma$ bounds. 

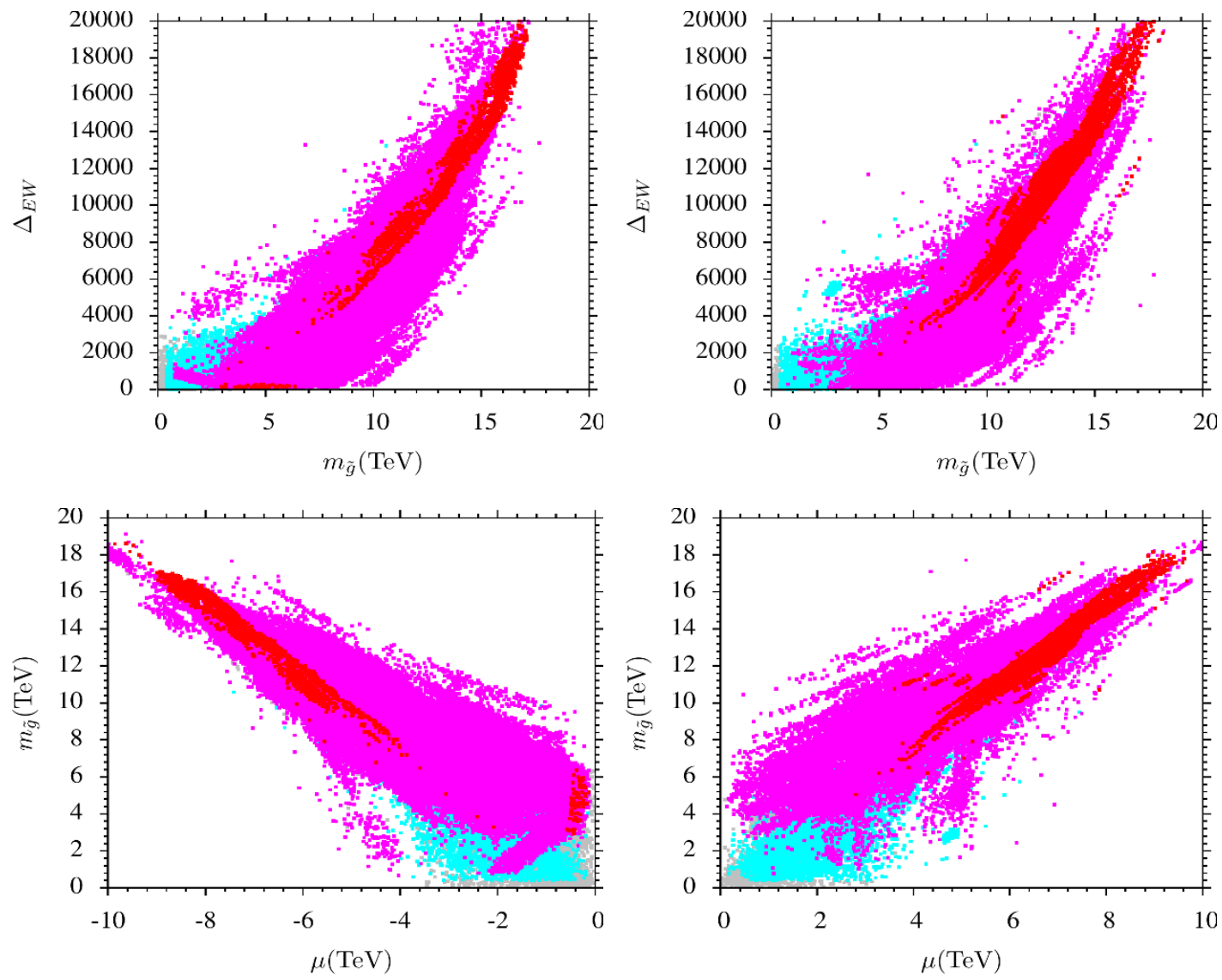

Figure 6. Plots in $\Delta_{E W}-m_{\tilde{g}}$ and $\mu-m_{\tilde{g}}$ planes. Color coding and panel description are same as in figure 1, except we do not apply gluino bounds mentioned in section 2 .

maximum value of $m_{L} \approx 10 \mathrm{TeV}$. In the $\tan \beta-m_{H_{u, d}}$ plane, right panel seems to be more populated in red points as compared to the left panel. We can see that in the right panel red points are $10 \lesssim \tan \beta \lesssim 60$ with $0 \lesssim m_{H_{u, d}} \lesssim 7 \mathrm{TeV}$. This apparent difference is due to lack of data in the case of $\mu<0$. By generating more data, we can reduce the apparent differences. In $\tan \beta-A_{0}$ plane too, we see the same situation. But one thing is clear from both panels which is that data favours $A_{0}<0$.

Plots in $\mu-\Delta_{E W}$ and $\Delta_{H S}-\Delta_{E W}$ planes are shown in figure 4. Color coding is same as in figure 1. The top left and right panels depict plots with large ranges of parameters as compared to the bottom left and right panels. Moreover, the left and right panels represent $\mu<0$ and $\mu>0$ scenario, respectively. With large parameter ranges, the top two panels almost look like the mirror images of each other. But from the left panel we see that it is relatively easy to have WMAP9 compatible red points with $\mu<0$ as compared right panel with $\mu>0$ where the minimal value of $\Delta_{E W}$ for red points is about 2800 . In order to investigate further we redraw the same plot with small ranges of parameters. We immediately note that there are some red points below $\Delta_{E W} \lesssim 200$. We also note that the minimal values of $\Delta_{E W}$ with and without WMAP9 bounds are $56(1.78 \% \mathrm{FT})$ and $24(4.1 \%$ 

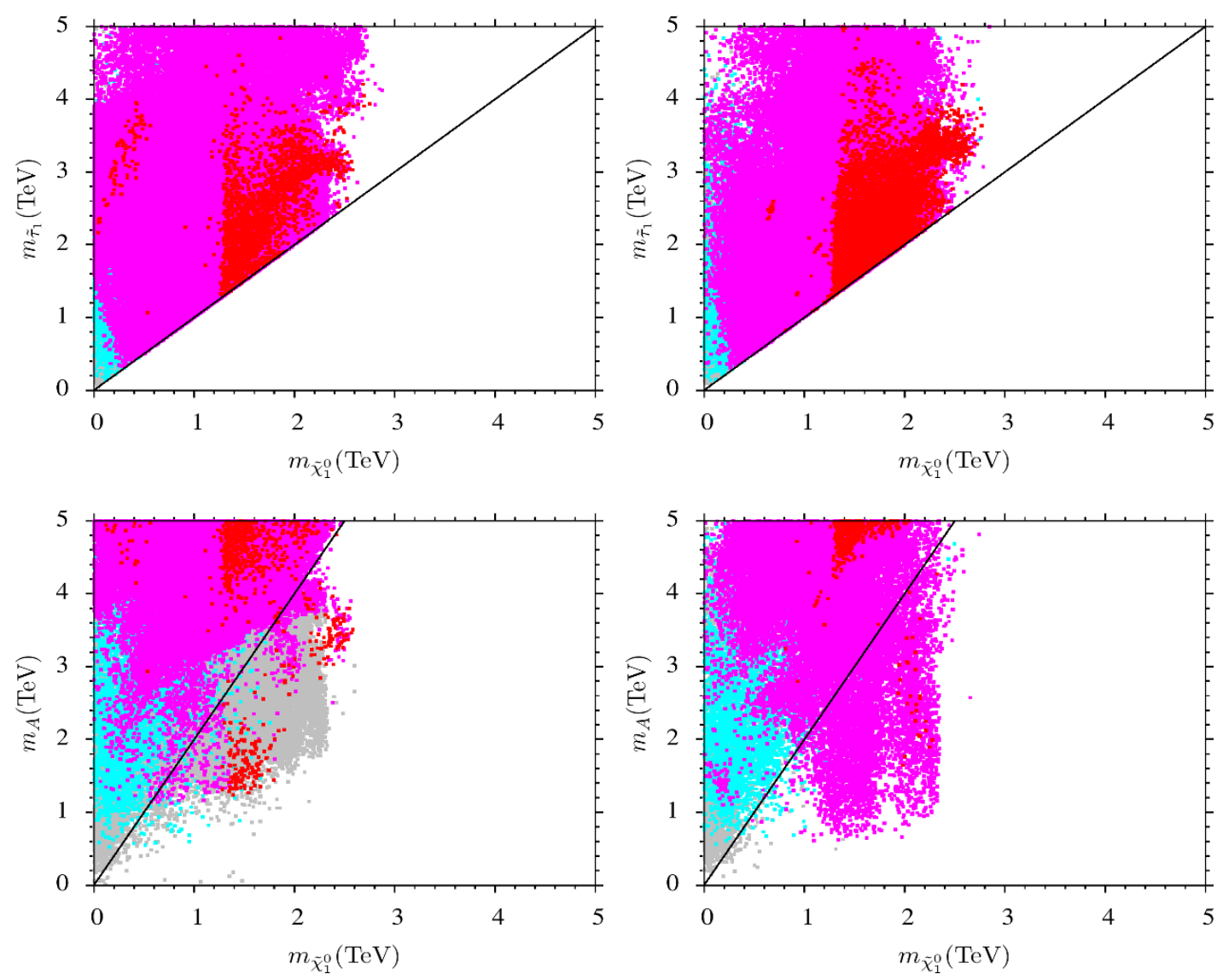

Figure 7. Plots in $m_{\tilde{\chi}_{1}^{0}}-m_{\tilde{\tau}}$ and $m_{\tilde{\chi}_{1}^{0}}-m_{A}$ planes. Color coding and panel description are same as in figure 1.

FT) respectively. On the other hand in the right panel we see that the minimum value of $\Delta_{E W}$ for magenta points is $31(3.2 \% \mathrm{FT})$. We have also checked that in the right panel, points with relatively small values of $\Delta_{E W}$ have relic density of about 1 . This shows that if we try more harder we can get some solutions with small $\Delta_{E W}$ and compatible with the WMAP9 bounds. In the bottom left and right panels we show plots in $\Delta_{H S}-\Delta_{E W}$ plane. Here we see that for the entire data $\Delta_{H S} \gtrsim \Delta_{E W}$. We note that for $\mu<0$ case the minimal value of $\Delta_{H S}$ is $1125(0.08 \% \mathrm{FT})$ with $\Delta_{E W}$ value of $297(0.33 \% \mathrm{FT})$, while we have $963(0.1 \% \mathrm{FT})$ and $285(0.35 \% \mathrm{FT})$ for $\Delta_{H S}$ and $\Delta_{E W}$ respectively for $\mu>0$. It was shown in dedicated studies of natural supersymmetry [84, 85] that with the above definitions of $\Delta_{E W}$ and $\Delta_{H S}$ it is possible to have values for both the measures $\lesssim 50$ simultaneously.

In figure 5 we show graphs in $m_{h}-\Delta_{E W}, m_{h}-\mu$ and $m_{h}-m_{3 / 2}$ planes. Color coding is same as in figure 1 with only one exception that there are no magenta color points. In these plots vertical solid black lines represent Higgs mass bounds of $123 \mathrm{GeV}$ and $127 \mathrm{GeV}$. Here we want to show $\Delta_{E W}, \mu, m_{3 / 2}$ and corresponding $m_{h}$ values. We note that most of our solutions in both the left and right panels are around $m_{h} \approx 125 \mathrm{GeV}$. Plots in $m_{h}-m_{3 / 2}$ show that the gravitino mass $m_{3 / 2}$ has to be more than $2 \mathrm{TeV}$ in both cases to have solutions consistent with bounds on Higgs mass 123 to $127 \mathrm{GeV}$. 

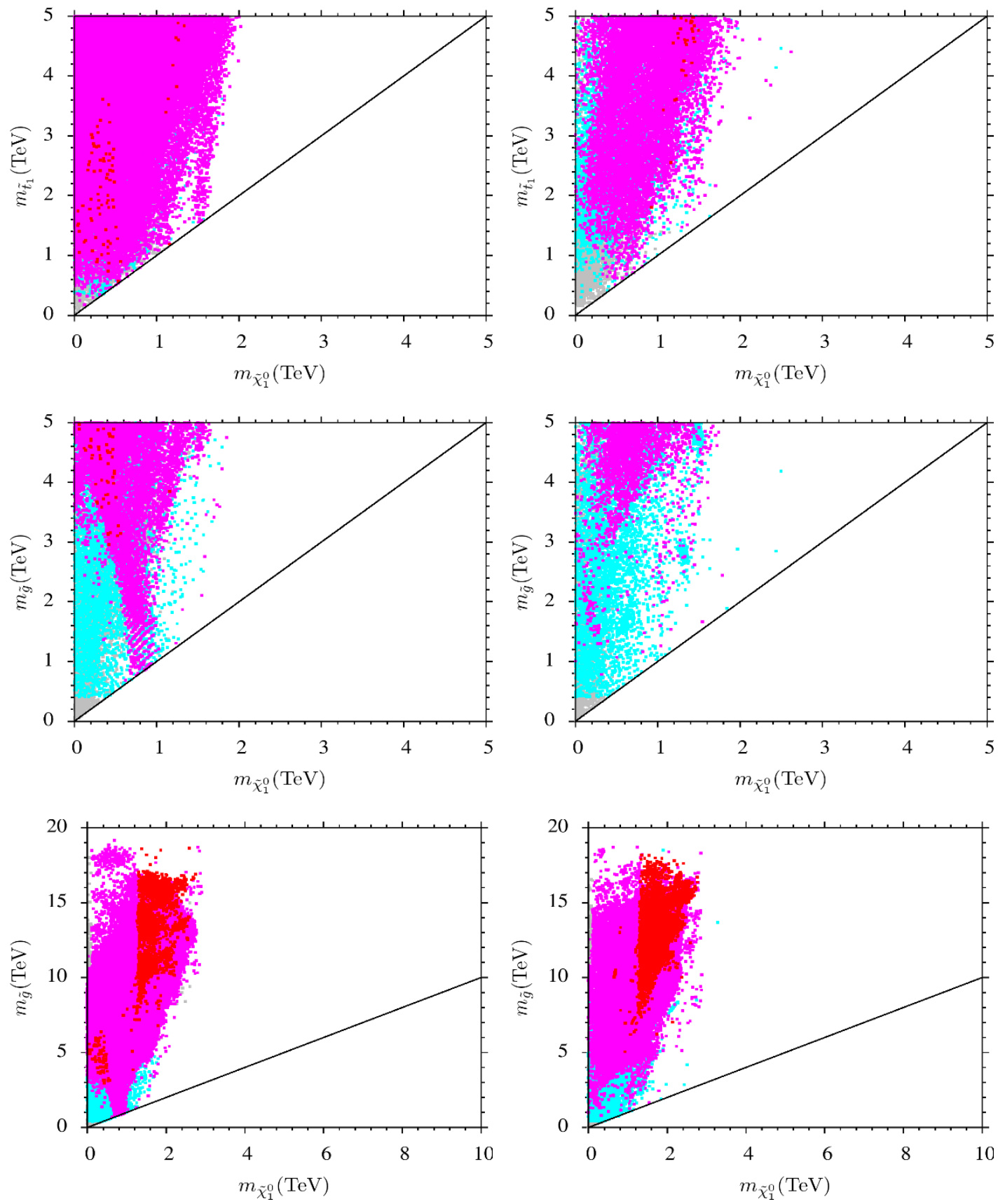

Figure 8. Plots in $m_{\tilde{\chi}_{1}^{0}}-m_{\tilde{t}_{1}}$ and $m_{\tilde{\chi}_{1}^{0}}-m_{\tilde{g}}$ planes. Color coding and panel description are same as in figure 1, except in middle and bottom panels we do not apply gluino bounds mentioned in section 2 . 

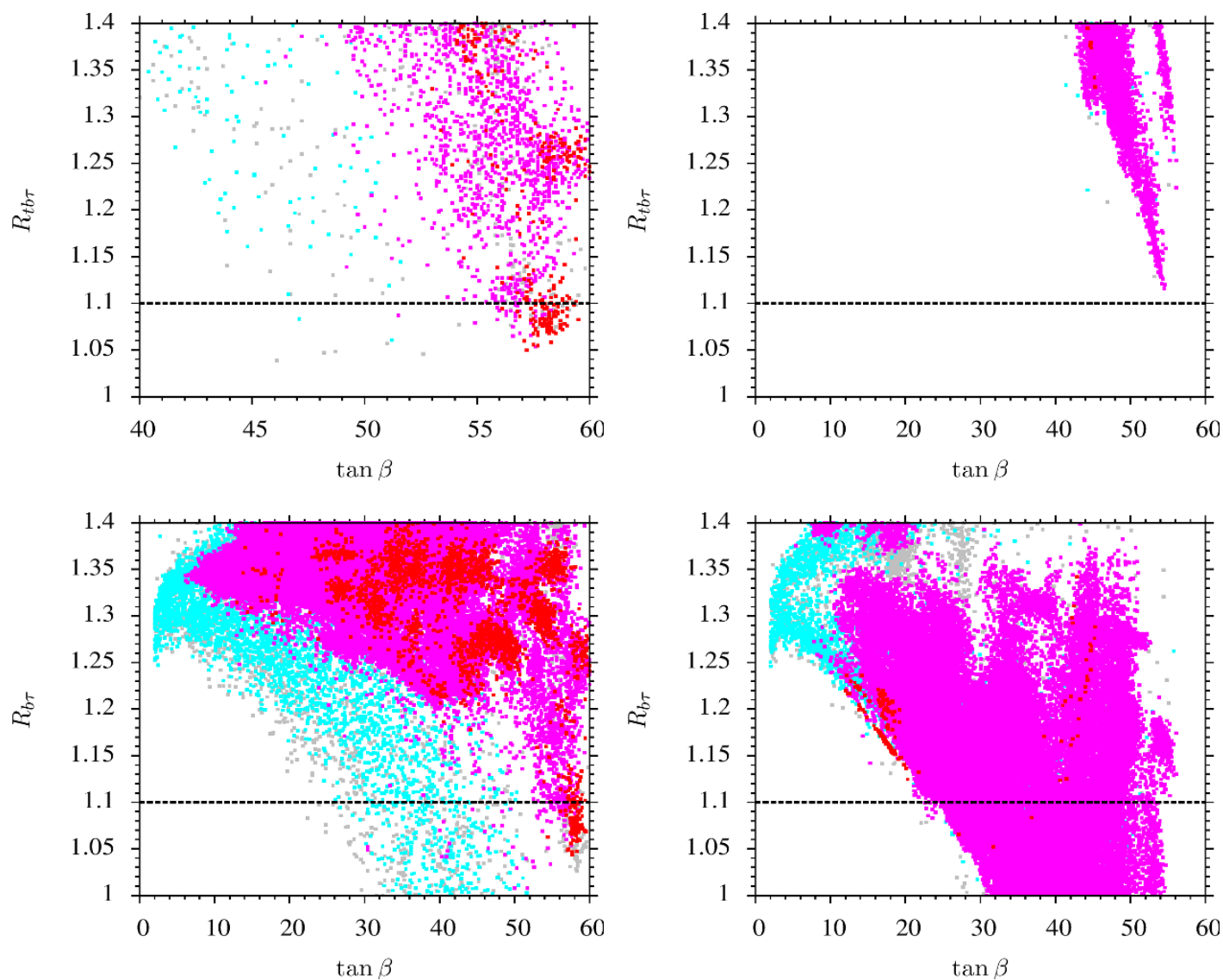

Figure 9. Plots in $\tan \beta-R_{t b \tau}$ and $\tan \beta-R_{b \tau}$ planes. Color coding and panel description are same as in figure 1.
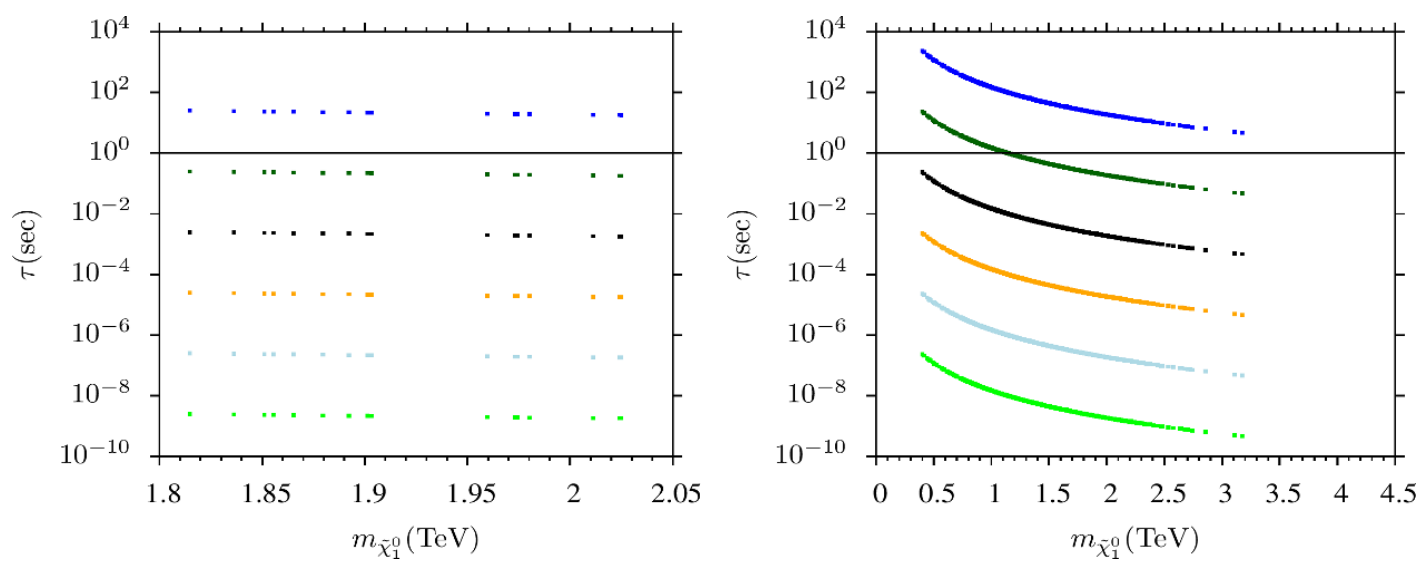

Figure 10. The NLSP bino-like neutralino mass $\left(m_{\tilde{\chi}_{1}^{0}}\right)$ versus lifetime $(\tau)$ plot. We use $C_{a Y Y}=$ $8 / 3, N=6$. Here, light-Green, light-blue, orange, black, dark-green, and blue colors represent $f_{a}=$ $10^{10}-10^{15} \mathrm{GeV}$, respectively. The black solid line shows $\tau=1$ second. 

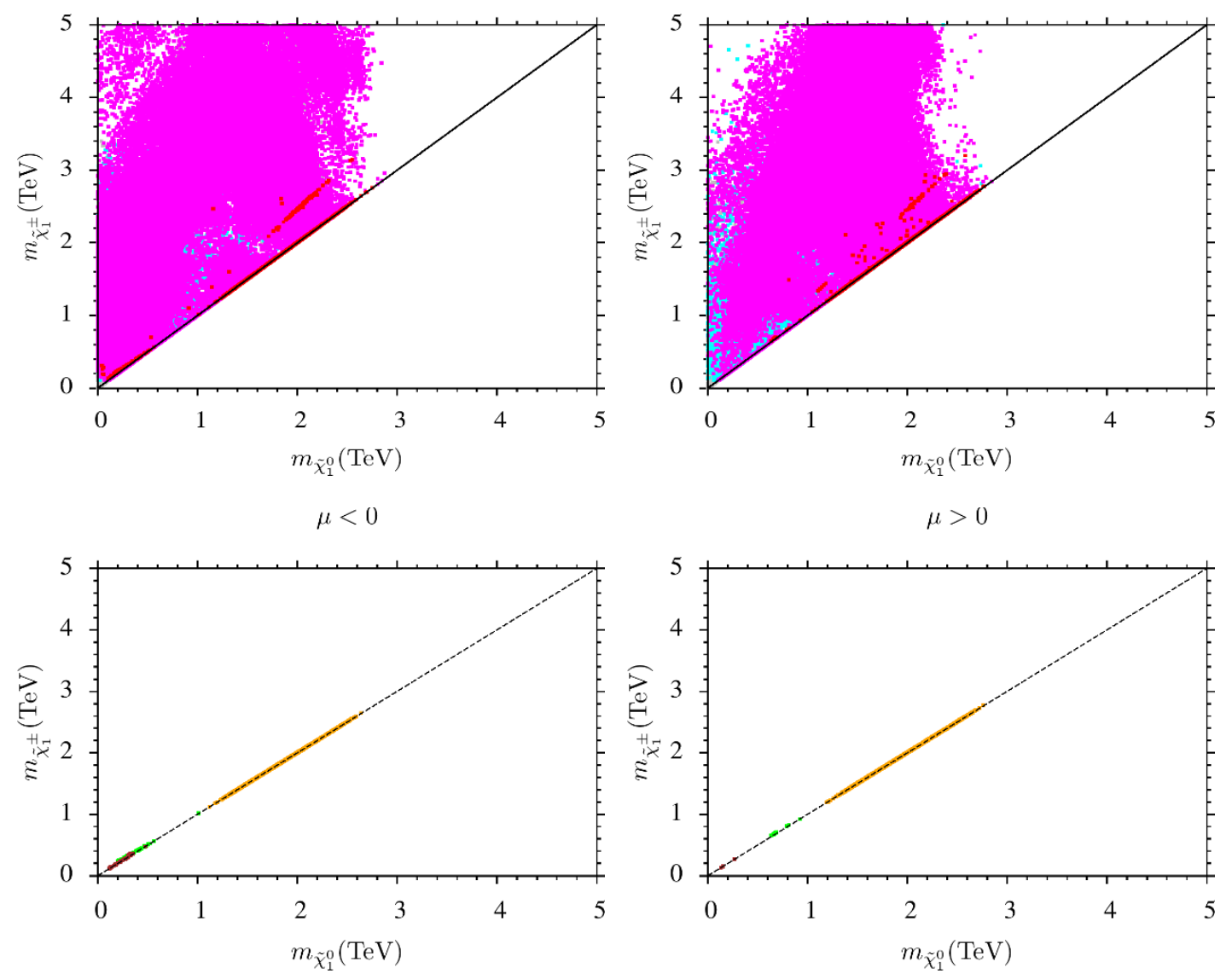

Figure 11. Plots in $m_{\tilde{\chi}_{1}^{0}}-m_{\tilde{\chi}_{1}^{ \pm}}$planes. Color coding and panel description are same as in figure 1 for plots in top left and right panels. In bottom left and right plots, the brown, green and orange points represent higgsino-like, bino-like, and wino-like neutralinos respectively with same panel description as in top panels.

We know that the LHC is a color particle producing machine. Among the color particles, gluinos are the smoking guns for the SUSY signals. Recent analysis have put limits of gluino mass $m_{\tilde{g}} \gtrsim 1.7 \mathrm{TeV}$ (for $m_{\tilde{g}} \sim m_{\tilde{q}}$ ) and $m_{\tilde{g}} \gtrsim 1.3 \mathrm{TeV}$ (for $m_{\tilde{g}} \gtrsim m_{\tilde{q}}$ ) [48, 49]. In figure 6 we present plots in $m_{\tilde{g}}-\Delta_{E W}$ and $m_{\tilde{g}}-\mu$ planes. Color coding is same as in figure 1 except we do not apply gluino mass bounds mentioned in section 2 . The top left and right panels depict plots with large ranges of parameters as compared to the bottom left and right panels. Moreover, the left and right panels represent $\mu<0$ and $\mu>0$ scenario receptively. Here we show that in both scenarios we have heavy gluinos as $M_{3}$ is a free parameter in our model. Such solutions can easily evade the above mentioned LHC bounds on gluino and squarks. In top left frame, we see that we have $m_{\tilde{g}} \gtrsim 3 \mathrm{TeV}$ for small values of $\Delta_{E W}$ in case of red points. Interestingly, there exists a region of parameter space with $|\mu| \lesssim 500 \mathrm{GeV}$ and $\Delta_{E W} \lesssim 300$, where gluino masses are from 3 to $7 \mathrm{TeV}$, and the first two-generation squarks and sleptons are respectively in the mass ranges $[4,7] \mathrm{TeV}$ and $[2,4] \mathrm{TeV}$. Because such parameter space is natural from low-energy fine-tuning definition while the gluino and first two-generation squarks/sleptons can not be probed at the $14 \mathrm{TeV}$ LHC, this will 

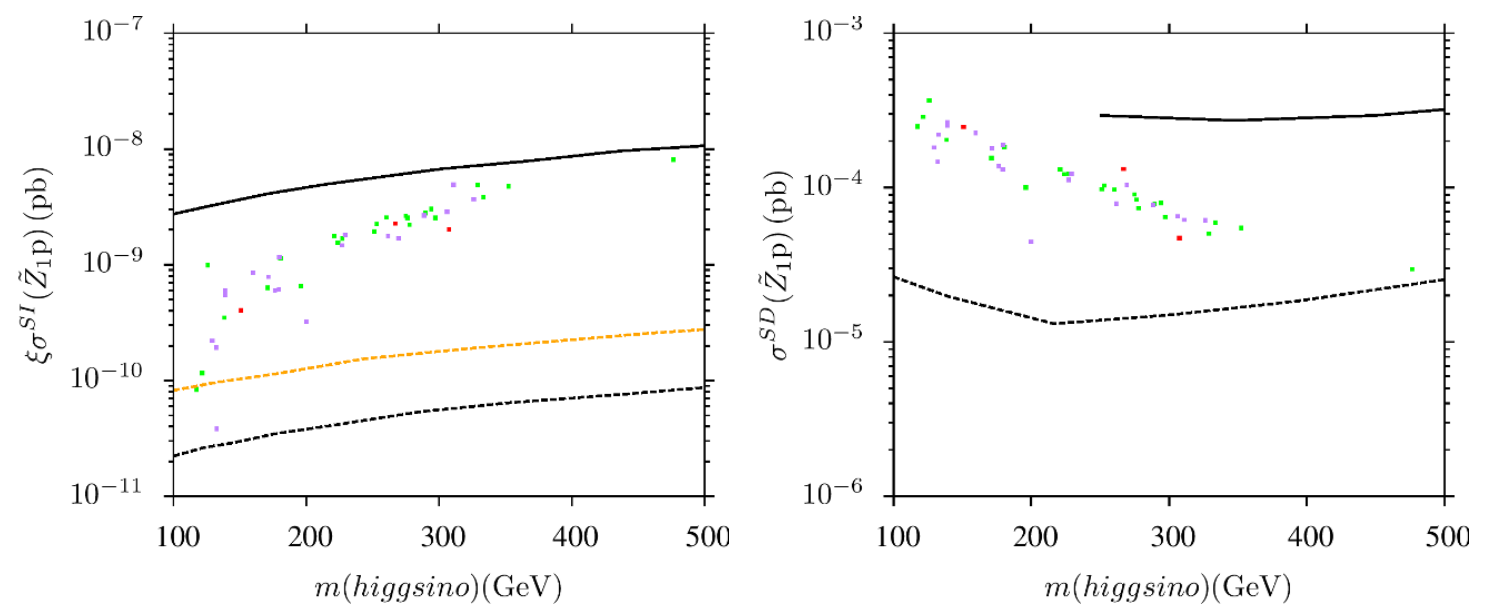

Figure 12. Plots in rescaled higgsino-like neutralino spin-independent cross section $\xi \sigma^{S I}\left(\tilde{Z}_{1} p\right)$ versus $m$ (higgsino) and (non-rescaled) higgsino-like neutralino spin-dependent cross section $\sigma^{S D}\left(\tilde{Z}_{1} p\right)$ versus $m$ (higgsino). In the left panel, the orange solid line represents the current upper bound set by the CDMS experiment and black solid line depicts the upper bound set by XENON100, while the orange and black dashed lines represent respectively the future reach of the SuperCDMS and XENON1T experiments. In the right panel, the IceCube DeepCore (black solid line) bound is shown and future IceCube DeepCore bound is depicted by the black dashed line. Green points represent solutions with $\Delta_{E W} \gtrsim 100$, purple points display solutions with $50 \lesssim \Delta_{E W} \lesssim 100$ while red solutions satisfy $\Delta_{E W} \lesssim 50$. Here, we have combined the solutions with $\mu<0$ and $\mu>0$.

provide a strong motivation for $33 \mathrm{TeV}$ and $100 \mathrm{TeV}$ proton-proton colliders. In the top right frame, we have red points around $m_{\tilde{g}} \sim 5 \mathrm{TeV}$ with $\Delta_{E W} \sim 2000$. Even if we consider magenta points, we see that we lose very tiny amount of data because of LHC bounds on gluino mass and most of our data remains intact. We also note that in our model $\Delta_{E W}$ can be small over the gluino mass range of 2 to $10 \mathrm{TeV}$ (magenta points). It is shown in [86] that the squarks/gluinos of $2.5 \mathrm{TeV}, 3 \mathrm{TeV}$ and $6 \mathrm{TeV}$ may be probed by the LHC14, High Luminosity (HL)LHC14 and High Energy (HE) LHC33, respectively. This clearly shows that our models have testable predictions. Moreover, in future if we have collider facility with even higher energy, we will be able to probe over even larger values of sparticle masses.

We present results with neutralino mass verses $\tilde{\tau}_{1}, A$ and $\tilde{\chi}_{1}^{ \pm}$masses in figure 7 . Color coding is same as in figure 1 and same panel description. Solid black lines are just to guide the eyes, where we can expect to have coannihilation and resonance solutions. In the top left and right panels we have plots in $m_{\tilde{\chi}_{1}^{0}}-m_{\tilde{\tau}_{1}}$ plane. We here note that in the left panel, if do not care about the stringent WMAP9 $5 \sigma$ bounds we have $\tilde{\tau}_{1}$ nearly degenerate with $\tilde{\chi}_{1}^{0}$ from $0.3 \mathrm{TeV}$ to $2.8 \mathrm{TeV}$. But the solutions satisfy WMAP9 $5 \sigma$ bounds have stau mass in the range of $1.2 \mathrm{TeV}$ to $2.8 \mathrm{TeV}$. In the right panel, stau mass range is $0.8 \mathrm{TeV}$ to $2.6 \mathrm{TeV}$ while we see the solutions without WMAP9 bonds have the have same stau mass range as we have in the left panel. Here we also note that the next to NLSP (NNLSP) $m_{\tilde{\chi}_{1}^{ \pm}}$is close to NLSP $m_{\tilde{\tau}}$ in mass. Their masses also lie within the $20 \%$ of LSP $\tilde{\chi}_{1}^{0}$ mass. In the bottom left and right panels of the figure we present plots in $m_{\tilde{\chi}_{1}^{0}}-m_{A}$ plane. We see that, in both 
panels we have A-resonance solutions for more than $1 \mathrm{TeV} m_{A}$ without WMAP9 bounds. But if WMAP9 $5 \sigma$ consistent points have $m_{A} \gtrsim 2 \mathrm{TeV}$.

Graphs in $m_{\tilde{\chi}_{1}^{0}}-m_{\tilde{t}_{1}}$ and $m_{\tilde{\chi}_{1}^{0}}-m_{\tilde{g}}$ planes are shown in figure 8 with the same color coding and panel description given in figure 1, except in middle and bottom panels we do not apply gluino bounds mentioned in section 2. From top left panel we see that we have two red points compatible with the WMAP9 bounds and representing neutralino-stop coannihilation scenario with mass around $570 \mathrm{GeV}$ and $1.2 \mathrm{TeV}$ respectively. On the other hand in the right panel we do not have red points along the line but we know that it is just because of lack of statistics (for studies on color and charge breaking vacua in the MSSM, see [87-89]). In the middle left and right panels we show graphs in $m_{\tilde{\chi}_{1}^{0}}-m_{\tilde{g}}$. In both cases we see that there are no WMAP9 compatible red points. But we do note that we have some magenta solutions where gluino and neutralino masses are almost degenerate and $\Omega h^{2}<1$. In the right panel we see only one magenta point near the black line but we can always generate more data around this point. Graphs in the bottom panels show that in our model, we can accommodate gluinos as heavy as $18 \mathrm{TeV}$ consistent with WMAP9 $5 \sigma$ bounds. Such a scenario suggest that there should be very high energy collider in order to probe such model points.

We quantify $t-b-\tau$ and $b-\tau$ the Yukawa coupling unification (YU) via the R-parameter

$$
R_{t b \tau} \equiv \frac{\max \left(y_{t}, y_{b}, y_{\tau}\right)}{\min \left(y_{t}, y_{b}, y_{\tau}\right)}, R_{b \tau} \equiv \frac{\max \left(y_{b}, y_{\tau}\right)}{\min \left(y_{b}, y_{\tau}\right)}
$$

where $y_{t}, y_{b}$ and $y_{\tau}$ are Yukawa couplings at the scale of the Grand Unified Theory (GUT). $R_{t b \tau}=1\left(R_{b \tau}=1\right)$ means $y_{t}=y_{b}=y_{\tau}\left(y_{b}=y_{\tau}\right)$ that is a solution with perfect $t-b-\tau(b-\tau)$ YU.

In figure 9 we present graphs in $\tan \beta-R_{t b \tau}$ and $\tan \beta-R_{b \tau}$ planes. Color coding is same as in figure 1. The left panels represent graphs in $\tan \beta-R_{t b \tau}$ and $\tan \beta-R_{b \tau}$ planes in case of $\mu<0$ and the right panels represent plots in $\tan \beta-R_{b \tau}$ and $\tan \beta-R_{t b \tau}$ for $\mu>0$. The horizontal black dashed line represents $10 \%$ or better $t-b-\tau(b-\tau)$ YU. We see in the top left panel that in our scans we have $10 \%$ or better YU solutions for $\tan \beta \sim 50-60$. The minimal value for $R_{t b \tau}$ we have is 1.05 ( $5 \% \mathrm{YU}$ ). We note that red points below the dashed line have $m_{h} \sim 125 \mathrm{GeV}$, gluino in the mass range of $8 \mathrm{TeV}$ to $10 \mathrm{TeV}$, the first two generation squarks and sleptons are in the mass ranges of $8 \mathrm{TeV}$ to $9.5 \mathrm{TeV}$ and $3 \mathrm{TeV} 3.5 \mathrm{TeV}$ respectively. The third generation squarks and sleptons lie in the mass ranges of $5 \mathrm{TeV}$ to $7.5 \mathrm{TeV}$ and $1.3 \mathrm{TeV}$ to $3 \mathrm{TeV}$. They also have large values for $|\mu|(\sim-5 \mathrm{TeV}$ to $-4 \mathrm{TeV})$ and $\Delta_{E W} \sim 4000$ to 8500 . More or less magenta points also have the similar mass spectrum. It was shown in more exhausted studies (see e.g [90-93] and references there in) with non-universal gaugino masses that one can have $100 \%$ YU with the LHC testable predictions.

In the bottom left panel we have $b-\tau$ YU solutions. Since this is a less constraint situation, we have $10 \%$ or better YU solutions for a wider range of $\tan \beta$, i.e., $30 \lesssim \tan \beta \lesssim$ 60. Here, the minimal value of $R_{b \tau}$ is about 1.04 (4\% YU). Moreover, the particle mass spectra also have slightly wider ranges as compared to $t-b-\tau$ YU case. We also note that those magenta points, which do not satisfy WMAP9 bounds, have more or less the same mass ranges as given above. 
In the top right panel, we see that we do not have even magenta solution with $10 \%$ or better $t-b-\tau$ YU with $\mu>0$. It was noticed that in a SUSY SO(10) GUT with non-universal SSB gaugino masses at $M_{\mathrm{GUT}}$ and $\mu>0, t-b-\tau$ Yukawa unification [94-96] can lead one to predict the lightest $\mathrm{CP}$ even Higgs boson mass to be $125 \mathrm{GeV}$ [97-99]. Even if we consider gaugino-universality $10 \%$ or better $t-b-\tau$ YU can be achieved consistent with the LHC bounds [100] but very hard to satisfy relic density bounds. In our scans, we have solutions with $12 \% t-b-\tau$ YU, if we do dedicated searches for better YU solutions, we can get them. Since here, we are not so keen to have $100 \%$ YU solutions but to give a flavor that our model can admit such solutions. In the bottom right panel we see that we have only three red points. On the other hand we have plenty of magenta points with $10 \%$ or better $b-\tau$ YU. In fact, we have $R_{b \tau}=1$, i.e., $100 \% b-\tau$ YU solutions for $\tan \beta \sim 30-55$. In order to save such solutions we have to add some extra physics to the MSSM. In such scenario we can consider SUSY models augmented with Peccei-Quinn(PQ) solution to the strong CP problem [101104] (PQMSSM). In SUSY context the axino field is just one element of an axion supermultiplet. The axion supermutiplet contains a complex scalar field, whose real part is the $R$-parity even saxion field $s(x)$ and whose imaginary part is the axion field $a(x)$. The supermutliplet also contains an $R$-parity odd spin half Majorana field, the axino $\tilde{a}(x)[105-107] .{ }^{6}$ In case where $\Omega h^{2} \gtrsim 1$, one way to have relic density within the observed range if we assume the $\tilde{\chi}_{1}^{0}$ may not be the LSP, but instead decays to much lighter state, such as $\tilde{\chi}_{1}^{0} \rightarrow \gamma \tilde{a}$,

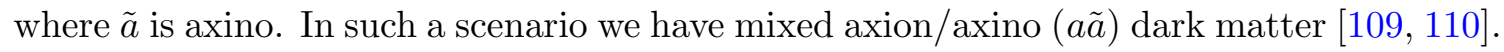
In this way the neutralino abundance is converted into an axino abundance with $[111,112]$

$$
\Omega_{\tilde{a}} h^{2}=\frac{m_{\tilde{a}}}{m_{\tilde{\chi}_{1}^{0}}} \Omega_{\tilde{\chi}_{1}^{0}}^{2} .
$$

It is important to know the life time $(\tau)$ of decaying neutralino. If it is more than 1 second, it can disturb Big Bang Nucleosynthesis (BBN) (see [113] and references there in). We first calculate $m_{\tilde{a}}$ for a given $m_{\tilde{\chi}_{1}^{0}}$ and its relic density $\Omega_{\tilde{\chi}_{1}^{0}}^{2}$ by assuming relic density of axino $\Omega_{\tilde{a}} h^{2}=0.11$ by using eq. (4.2). We then follow [114] to calculate the lifetime for the decaying NLSP neutralino. We use $C_{a Y Y}=8 / 3$ in the DFSZ model $[115,116], N=6$ (the color anomaly model dependent factor). We present our calculations in figure 10, where we display the NLSP bino-like neutralino mass $\left(m_{\tilde{\chi}_{1}^{0}}\right)$ versus its lifetime $(\tau)$. Panel description is same as in figure 1. Here points in various colors correspond to various choices of the axion decay constant $f_{a}$ values. The light-green, light-blue, orange, black, dark-green and blue represent $f_{a}=10^{10}-10^{15} \mathrm{GeV}$, respectively. The black solid line show $\tau=1$ second. In the left panel, the plot appears somewhat flat as compare to the right plot in right panel because of small mass range. From both the frames, it is clearly visible that for $f_{a}=10^{15} \mathrm{GeV}$, life time of NLSP bino is more than 1 second. For $f_{a}=10^{14} \mathrm{GeV}$, in the right panel, points with bino mass greater than $1 \mathrm{TeV}$ are allowed.

In another approach to reduce relic density is to assume the additional late decaying scalar fields are present in the model. These fields may get produced at large rates via coherent oscillations. If they temporarily dominate the energy density of the Universe, and then decay to mainly SM particles, they may inject considerable entropy into the cosmic

\footnotetext{
${ }^{6}$ For a review see e.g. [108].
} 
soup, thus diluting all relics which are present at the time of decay. Entropy injection can occur at large rates for instance from saxion production in the PQMSSM [117-125], or from moduli production and decay, as is expected in string theory [126-132]. However, it was shown in [133] that the efforts to dilute the relic density of neutralino below the observed dark matter relic density through entropy injection from saxion decays such as saxion decays to gluon violate the CMB bound on $\Delta N_{\text {eff }}$, where $\Delta N_{\text {eff }}$ is the apparent number of additional effective neutrinos.

On the other hand, the solutions with good YU may also have small relic density $\Omega h^{2} \sim 10^{-5}-10^{-2}$. In such cases the neutralino abundance can be augmented in the PQMSSM case where $m_{\tilde{a}}>m_{\tilde{\chi}_{1}^{0}}$ and additional neutralinos are produced via thermal axino production and decay $m_{\tilde{a}} \rightarrow m_{\tilde{\chi}_{1}^{0}} \gamma$ [121-125]. In these cases, the CDM tends to be neutralino dominated with a small component of axions.

In figure 11 we show graphs in $m_{\tilde{\chi}_{1}^{0}}-m_{\tilde{\chi}_{1}^{ \pm}}$plane with the same panel description as in figure 1. The top left and right frames have same color coding as in figure 1. From these frames, it is apparent that we have solutions from $0.1 \mathrm{TeV}$ to $2.8 \mathrm{TeV}$. In bottom frames we further analyse these points on the basis of neutralino composition. Here orange, green and brown points represent neutralino with more than $90 \%$ wino, more than $80 \%$ bino and more than $50 \%$ higgsino composition, respectively. It is to be noted that orange and the green points satisfy all constraints given section 2 but brown point do not satisfy relic density bounds. Here, we want to show that in our scans where the neutralino and chargino masses are almost degenerate, and neutralino LSP can be of bino, wino and higgsino like. We immediately see that in both cases $(\mu<0$ and $\mu>0)$, wino-type neutralino have masses more than $1 \mathrm{TeV}$. On the other hand bino-like solutions have masses less than $1 \mathrm{TeV}$ while higgsino-type solutions have mass range of 150 to $600 \mathrm{GeV}$. It is shown in $[50,51]$ that for NFW and Einasto distribution, the entire mass range of thermal wino dark matter from 0.1 to $3 \mathrm{TeV}$ may be excluded. In a recent study [134], wino as dark matter candidate is excluded in the mass range bellow $800 \mathrm{GeV}$ from antiproton and between $1.8 \mathrm{TeV}$ to $3.5 \mathrm{TeV}$ from the absence of a $\gamma$-ray line feature toward the galactic center. Since our bino-like points have some admixture of higgsinos and that is why they have large nucleon-neutralino scattering cross section. Such solutions are also under stress because of the current upper bound set by XENON100 [135]. Here, we argue that such wino-like (bino-like) neutralino solutions may avoid the above mentioned bounds. For example, the wino-like neutralino density is smaller than the observed density. Otherwise, instead of treating them as the LSPs we assume that they are the NLSP and may decay to axino and $\gamma$ as we have discussed above. Similarly, we can also assume the mechanism of late decaying fields via coherent oscillations or production of moduli and their decay as we argued previously. In addition to it, we can also invoke $R$-parity violation scenario, where the bino LSP and similarly winolike neutralino can decay to the SM fermions via sfermion exchange. In order to address the issue of underabundance of higgsino-like solutions we argue that mainly higgsino-like neutralino by itself does not make a good cold dark matter candidate and we need additional dark matter candidates to match the observed dark matter relic density. For this purpose we assume that the higgsino could make up only a fraction of the relic dark matter and the remaining abundance is comprised of axions produces through the vacuum misalignment 
mechanism [137-141]. This is why we could expect the higgsino relic density somewhat suppressed between $1-15$ in the present universe. This not only provides us with the opportunity to look for higgsinos, despite the fact that they would only constitute a fraction of the measured relic dark matter abundance but also the possibility to detect axions. We would also like to mention that our higgsino-like solutions especially for $\Delta_{E W} \lesssim 50$ more or less look like the solutions form radiative natural SUSY [142]. Since such solutions tend to have large direct and indirect neutralino detection rates, let us check the status of our higgsino-like solutions. We will follow [143]. In the left panel of figure 12 we plot rescaled higgsino-like neutralino spin-independent cross section $\xi \sigma^{S I}\left(\tilde{Z}_{1} p\right)$ versus $m$ (higgsino) (in this figure for both panels we have combined solutions with $\mu<0$ and $\mu>0$ ). The orange solid line represents the current upper bound set by the CDMS experiment and black solid line depicts upper bound set by XENON100 [135], while the orange (black) dashed line represents future reach of SuperCDMS [144] (XENON1T [145]). We rescale our result by a factor $\xi=\Omega_{\tilde{Z}_{1}} h^{2} / 0.11$ in order to account for the fact that the local relic density might be much less than the usually assumed value $\rho_{\text {local }} \simeq 0.3 \mathrm{GeV} / \mathrm{cm}^{3}$ as pointed out in [146]. Here, we see that all the points lie below the current upper bounds set by CDMS XENON100 experiments. It is very clear that the future experiments like XENON1T will be able to probe almost all of our model points. This shows our results are in agreement with [143] where it was shown that all higgsino points could be tested by the XENON1T and one could discover neutralino (WIMPs) or exclude the concept of electroweak naturalness in $R$-parity conserving natural SUSY models. In right panel of figure 12 , we have a plot of (non-rescaled) higgsino-like neutralino spin-dependent cross section $\sigma^{S D}\left(\tilde{Z}_{1} p\right)$ versus $m$ (higgsino). The IceCube DeepCore and future IceCube DeepCore bounds are shown in black solid line and black dashed line [147]. Color coding is same as in left panel. Here we do not rescale our results because the IceCube detection depends on whether the Sun has equilibrated its core abundance between capture rate and annihilate rate [148]. It was shown in [149] that for the Sun, equilibrium is reached for almost all of SUSY parameter space. In this plot we see that the future IceCube DeepCore searches will be able to probe our entire set of solutions in our present scans.

In tables $1-3$, we list benchmark points for $\mu<0$ case. All of these points satisfy the sparticle mass, B-physics and Higgs mass constraints described in section 2. In table 1, point 1(2) represents the minimal value of $\Delta_{E W}$ not consistent and consistent with WMAP9 $5 \sigma$ bounds, while points 3-5 respectively correspond to the minimal value of $\Delta_{H S}$, best point with $t-b-\tau$ and $b-\tau$ YU, an example of heavy gluino solution. Points 3 and 4 also satisfy WMAP9 $5 \sigma$ bounds. In table 2, points 1, 2, 3 and 4 display neutralino-stau, neutralinostop, $m_{A}$-resonance and neutralino-gluino coannihilation, respectively. Point 4 is the case where relic density is below WMAP9 $5 \sigma$ bounds. In table 3 , point 1 represents bino-like neutralino, point 2 displays higgsino like neutralino, point 3 and point 4 are examples of wino-like neutralino. Point 2 and point 4 do not satisfy WMAP9 $5 \sigma$ bounds.

In tables $4-6$, we display benchmark points for $\mu>0$ case consistent with the sparticle mass, B-physics and Higgs mass constraints described in section 2. In table 4, points 1-4 respectively correspond to the minimal value of $\Delta_{E W}$, minimal value of $\Delta_{H S}$, best point with $b-\tau$ YU, an example of heavy gluino solution. Points 3 and 4 also satisfy WMAP9 $5 \sigma$ bounds. Table 5 and table 6 have similar description as table 2 and table 3. 


\section{Discussions and conclusion}

The three-family Pati-Salam models have been constructed systematically in Type IIA string theory on the $\mathbf{T}^{\mathbf{6}} /\left(\mathbb{Z}_{\mathbf{2}} \times \mathbb{Z}_{\mathbf{2}}\right)$ orientifold with intersecting D6-branes [23]. It was found that one model has a realistic phenomenology [44, 45]. Considering the Higgs boson mass around $125 \mathrm{GeV}$ and the LHC supersymmetry search constraints, we have revisited this three-family Pati-Salam model in details. We systematically scanned the viable parameter space for $\mu<0$ and $\mu>0$, and found that in general the gravitino mass is heavier than about $2 \mathrm{TeV}$ for both cases because of the Higgs boson mass low bound $123 \mathrm{GeV}$. In particular, we identified a natural region of parameter space where the electroweak finetuning can be as small as $\Delta_{E W} \sim 24-32$ (3-4\%). Also, we found another interesting region of parameter space with $|\mu| \lesssim 500 \mathrm{GeV}$ and $\Delta_{E W} \lesssim 300$, where the mass ranges for the gluino, and first two-generation squarks and sleptons are $[3,7] \mathrm{TeV},[4,7] \mathrm{TeV}$, and $[2,4] \mathrm{TeV}$, respectively. This will provide a strong motivation for $33 \mathrm{TeV}$ and $100 \mathrm{TeV}$ proton-proton colliders since it is natural from low-energy fine-tuning definition while the gluino and first two generation squarks/sleptons are heavy. In the whole viable parameter space which is consistent with all the current experimental constraints including the dark matter relic density bounds, the gluino mass range is $[3,18] \mathrm{TeV}$, the first two-family squarks have masses from 3 to $16 \mathrm{TeV}$, and the first two-family sleptons have masses from 2 to $7 \mathrm{TeV}$. Thus, the viable parameter space with heavy gluino and squarks is even out of reach of the $100 \mathrm{TeV}$ proton collider [150]. On the other hand, for the third-family sfermions, the NLSP light stop satisfying $5 \sigma$ WMAP bounds is in the mass range $[0.5,1.2] \mathrm{TeV}$, and the light stau can be as light as $800 \mathrm{GeV}$. We also showed various coannihilation and resonance scenarios through which the observed dark matter relic density can be achieved. Interestingly, the certain portions of parameter space have excellent $t-b-\tau$ and $b-\tau$ Yukawa coupling unification. Also, we highlighted the regions of parameter space where the LSP neutralino can be a bino, wino, or higgsino. We discussed various scenarios in which such solutions may avoid recent astrophysical bounds in case if they satisfy or above the correct dark matter relic density bounds. Prospects of finding higgsino-like neutralino in direct and indirect searches were shown and discussed as well. To be concrete, we displayed six benchmark tables depicting various interesting features of our model. Furthermore, because the LSP neutralino can be heavier than $1 \mathrm{TeV}$ and up to about $2.8 \mathrm{TeV}$, how to test such scenario at the $14 \mathrm{TeV}$ LHC is still a big question. Therefore, the $33 \mathrm{TeV}$ and $100 \mathrm{TeV}$ proton-proton colliders are indeed needed to probe our D-brane model.

\section{Acknowledgments}

We would like to thank Howard Baer, Eung-Jin Chun, Bin He, and Azar Mustafayev very much for helpful discussions. The work of TL, SR and XW is supported in part by by the Natural Science Foundation of China under grant numbers 10821504, 11075194, 11135003, and 11275246, and by the National Basic Research Program of China (973 Program) under grant number 2010CB833000. And the work of DVN was supported in part by the DOE grant DE-FG03-95-ER-40917. 
Open Access. This article is distributed under the terms of the Creative Commons Attribution License (CC-BY 4.0), which permits any use, distribution and reproduction in any medium, provided the original author(s) and source are credited.

\section{References}

[1] W. Buchmüller, K. Hamaguchi, O. Lebedev and M. Ratz, Supersymmetric standard model from the heterotic string, Phys. Rev. Lett. 96 (2006) 121602 [hep-ph/0511035] [INSPIRE].

[2] O. Lebedev et al., A mini-landscape of exact MSSM spectra in heterotic orbifolds, Phys. Lett. B 645 (2007) 88 [hep-th/0611095] [INSPIRE].

[3] J.E. Kim and B. Kyae, Flipped $\mathrm{SU}(5)$ from $Z_{12-I}$ orbifold with Wilson line, Nucl. Phys. B 770 (2007) 47 [hep-th/0608086] [INSPIRE].

[4] J.E. Kim and B. Kyae, Kaluza-Klein masses in nonprime orbifolds: $Z_{12-I}$ compactification and threshold correction, Phys. Rev. D 77 (2008) 106008 [arXiv:0712.1596] [INSPIRE].

[5] J.-H. Huh, J.E. Kim and B. Kyae, $\mathrm{SU}(5)_{\text {flip }} \times \mathrm{SU}(5)^{\prime}$ from $Z_{12-I}$, Phys. Rev. D 80 (2009) 115012 [arXiv: 0904.1108] [INSPIRE].

[6] V. Braun, Y.-H. He, B.A. Ovrut and T. Pantev, A heterotic standard model, Phys. Lett. B 618 (2005) 252 [hep-th/0501070] [INSPIRE].

[7] V. Braun, Y.-H. He, B.A. Ovrut and T. Pantev, The exact MSSM spectrum from string theory, JHEP 05 (2006) 043 [hep-th/0512177] [INSPIRE].

[8] V. Bouchard and R. Donagi, An SU(5) heterotic standard model, Phys. Lett. B 633 (2006) 783 [hep-th/0512149] [INSPIRE].

[9] I. Antoniadis, J.R. Ellis, J.S. Hagelin and D.V. Nanopoulos, GUT model building with fermionic four-dimensional strings, Phys. Lett. B 205 (1988) 459 [INSPIRE].

[10] I. Antoniadis, J.R. Ellis, J.S. Hagelin and D.V. Nanopoulos, An improved SU(5) $\times$ U(1) model from four-dimensional string, Phys. Lett. B 208 (1988) 209 [Addendum ibid. B 213 (1988) 562] [INSPIRE].

[11] I. Antoniadis, J.R. Ellis, J.S. Hagelin and D.V. Nanopoulos, The flipped $\mathrm{SU}(5) \times \mathrm{U}(1)$ string model revamped, Phys. Lett. B 231 (1989) 65 [INSPIRE].

[12] A.E. Faraggi, D.V. Nanopoulos and K.-J. Yuan, A standard like model in the $4 D$ free fermionic string formulation, Nucl. Phys. B 335 (1990) 347 [InSPIRE].

[13] I. Antoniadis, G.K. Leontaris and J. Rizos, A three generation $\mathrm{SU}(4) \times O(4)$ string model, Phys. Lett. B 245 (1990) 161 [INSPIRE].

[14] J.L. Lopez, D.V. Nanopoulos and K.-J. Yuan, The search for a realistic flipped SU(5) string model, Nucl. Phys. B 399 (1993) 654 [hep-th/9203025] [INSPIRE].

[15] D.V. Nanopoulos, Fenomenology, hep-ph/0211128 [INSPIRE].

[16] G.B. Cleaver, A.E. Faraggi, D.V. Nanopoulos and J.W. Walker, Phenomenology of non-Abelian flat directions in a minimal superstring standard model, Nucl. Phys. B 620 (2002) 259 [hep-ph/0104091] [INSPIRE].

[17] M. Berkooz, M.R. Douglas and R.G. Leigh, Branes intersecting at angles, Nucl. Phys. B 480 (1996) 265 [hep-th/9606139] [INSPIRE]. 
[18] L.E. Ibáñez, F. Marchesano and R. Rabadán, Getting just the standard model at intersecting branes, JHEP 11 (2001) 002 [hep-th/0105155] [INSPIRE].

[19] R. Blumenhagen, B. Körs, D. Lüst and T. Ott, The standard model from stable intersecting brane world orbifolds, Nucl. Phys. B 616 (2001) 3 [hep-th/0107138] [INSPIRE].

[20] M. Cvetič, G. Shiu and A.M. Uranga, Three family supersymmetric standard-like models from intersecting brane worlds, Phys. Rev. Lett. 87 (2001) 201801 [hep-th/0107143] [INSPIRE].

[21] M. Cvetič, G. Shiu and A.M. Uranga, Chiral four-dimensional $N=1$ supersymmetric type IIA orientifolds from intersecting D6 branes, Nucl. Phys. B 615 (2001) 3 [hep-th/0107166] [INSPIRE].

[22] M. Cvetič, I. Papadimitriou and G. Shiu, Supersymmetric three family SU(5) grand unified models from type IIA orientifolds with intersecting D6-branes, Nucl. Phys. B 659 (2003) 193 [Erratum ibid. B 696 (2004) 298] [hep-th/0212177] [INSPIRE].

[23] M. Cvetič, T. Li and T. Liu, Supersymmetric Pati-Salam models from intersecting D6-branes: a road to the standard model, Nucl. Phys. B 698 (2004) 163 [hep-th/0403061] [INSPIRE].

[24] M. Cvetič, P. Langacker, T.-J. Li and T. Liu, D6-brane splitting on type IIA orientifolds, Nucl. Phys. B 709 (2005) 241 [hep-th/0407178] [INSPIRE].

[25] M. Cvetič, T. Li and T. Liu, Standard-like models as type IIB flux vacua, Phys. Rev. D 71 (2005) 106008 [hep-th/0501041] [INSPIRE].

[26] C.-M. Chen, G.V. Kraniotis, V.E. Mayes, D.V. Nanopoulos and J.W. Walker, A supersymmetric flipped SU(5) intersecting brane world, Phys. Lett. B 611 (2005) 156 [hep-th/0501182] [INSPIRE].

[27] C.-M. Chen, G.V. Kraniotis, V.E. Mayes, D.V. Nanopoulos and J.W. Walker, A k-theory anomaly free supersymmetric flipped $\mathrm{SU}(5)$ model from intersecting branes, Phys. Lett. B 625 (2005) 96 [hep-th/0507232] [INSPIRE].

[28] C.-M. Chen, T. Li and D.V. Nanopoulos, Standard-like model building on type II orientifolds, Nucl. Phys. B 732 (2006) 224 [hep-th/0509059] [INSPIRE].

[29] R. Blumenhagen, M. Cvetič, P. Langacker and G. Shiu, Toward realistic intersecting D-brane models, Ann. Rev. Nucl. Part. Sci. 55 (2005) 71 [hep-th/0502005] [inSPIRE].

[30] G. Honecker, M. Ripka and W. Staessens, The importance of being rigid: D6-brane model building on $T^{6} / Z_{2} \times Z_{6}^{\prime}$ with discrete torsion, Nucl. Phys. B 868 (2013) 156 [arXiv:1209.3010] [INSPIRE].

[31] G. Honecker and T. Ott, Getting just the supersymmetric standard model at intersecting branes on the $Z_{6}$ orientifold, Phys. Rev. D 70 (2004) 126010 [Erratum ibid. D 71 (2005) 069902] [hep-th/0404055] [INSPIRE].

[32] G. Honecker, Chiral supersymmetric models on an orientifold of $Z_{4} \times Z_{2}$ with intersecting D6-branes, Nucl. Phys. B 666 (2003) 175 [hep-th/0303015] [INSPIRE].

[33] T.P.T. Dijkstra, L.R. Huiszoon and A.N. Schellekens, Chiral supersymmetric standard model spectra from orientifolds of Gepner models, Phys. Lett. B 609 (2005) 408 [hep-th/0403196] [INSPIRE]. 
[34] T.P.T. Dijkstra, L.R. Huiszoon and A.N. Schellekens, Supersymmetric standard model spectra from RCFT orientifolds, Nucl. Phys. B 710 (2005) 3 [hep-th/0411129] [INSPIRE].

[35] C. Vafa, Evidence for F-theory, Nucl. Phys. B 469 (1996) 403 [hep-th/9602022] [INSPIRE].

[36] R. Donagi and M. Wijnholt, Model building with F-theory, Adv. Theor. Math. Phys. 15 (2011) 1237 [arXiv: 0802.2969] [INSPIRE].

[37] C. Beasley, J.J. Heckman and C. Vafa, GUTs and exceptional branes in F-theory - I, JHEP 01 (2009) 058 [arXiv:0802.3391] [INSPIRE].

[38] C. Beasley, J.J. Heckman and C. Vafa, GUTs and exceptional branes in F-theory - II: experimental predictions, JHEP 01 (2009) 059 [arXiv:0806.0102] [INSPIRE].

[39] R. Donagi and M. Wijnholt, Breaking GUT groups in F-theory, Adv. Theor. Math. Phys. 15 (2011) 1523 [arXiv: 0808.2223] [INSPIRE].

[40] A. Font and L.E. Ibáñez, Yukawa structure from U(1) fluxes in F-theory grand unification, JHEP 02 (2009) 016 [arXiv:0811.2157] [INSPIRE].

[41] J. Jiang, T. Li, D.V. Nanopoulos and D. Xie, F-SU(5), Phys. Lett. B 677 (2009) 322 [INSPIRE].

[42] J. Jiang, T. Li, D.V. Nanopoulos and D. Xie, Flipped $\mathrm{SU}(5) \times \mathrm{UX}(1)$ models from F-theory, Nucl. Phys. B 830 (2010) 195 [arXiv:0905.3394] [INSPIRE].

[43] T. Li, SU(5) and $\mathrm{SO}(10)$ models from F-theory with natural Yukawa couplings, Phys. Rev. D 81 (2010) 065018 [arXiv:0905.4563] [INSPIRE].

[44] C.-M. Chen, T. Li, V.E. Mayes and D.V. Nanopoulos, A realistic world from intersecting D6-branes, Phys. Lett. B 665 (2008) 267 [hep-th/0703280] [INSPIRE].

[45] C.-M. Chen, T. Li, V.E. Mayes and D.V. Nanopoulos, Towards realistic supersymmetric spectra and Yukawa textures from intersecting branes, Phys. Rev. D 77 (2008) 125023 [arXiv:0711.0396] [INSPIRE].

[46] ATLAS collaboration, Observation of a new particle in the search for the standard model Higgs boson with the ATLAS detector at the LHC, Phys. Lett. B 716 (2012) 1 [arXiv: 1207.7214] [INSPIRE].

[47] CMS collaboration, Observation of a new boson at a mass of $125 \mathrm{GeV}$ with the CMS experiment at the LHC, Phys. Lett. B 716 (2012) 30 [arXiv:1207.7235] [INSPIRE].

[48] CMS collaboration, Search for gluino mediated bottom- and top-squark production in multijet final states in pp collisions at 8 TeV, Phys. Lett. B 725 (2013) 243 [arXiv:1305.2390] [INSPIRE].

[49] ATLAS collaboration, Search for squarks and gluinos with the ATLAS detector in final states with jets and missing transverse momentum using $\sqrt{s}=8$ TeV proton-proton collision data, arXiv:1405.7875 [INSPIRE].

[50] J. Fan and M. Reece, In wino veritas? Indirect searches shed light on neutralino dark matter, JHEP 10 (2013) 124 [arXiv:1307.4400] [INSPIRE].

[51] T. Cohen, M. Lisanti, A. Pierce and T.R. Slatyer, Wino dark matter under siege, JCAP 10 (2013) 061 [arXiv:1307.4082] [INSPIRE].

[52] H. Baer, F.E. Paige, S.D. Protopopescu and X. Tata, ISAJET 7.48: a Monte Carlo event generator for $p p, \bar{p} p$ and $e^{+} e^{-}$reactions, hep-ph/0001086 [INSPIRE]. 
[53] J. Hisano, H. Murayama and T. Yanagida, Nucleon decay in the minimal supersymmetric SU(5) grand unification, Nucl. Phys. B 402 (1993) 46 [hep-ph/9207279] [INSPIRE].

[54] Y. Yamada, SUSY and GUT threshold effects in SUSY SU(5) models, Z. Phys. C 60 (1993) 83 [INSPIRE].

[55] J.L. Chkareuli and I.G. Gogoladze, Unification picture in minimal supersymmetric SU(5) model with string remnants, Phys. Rev. D 58 (1998) 055011 [hep-ph/9803335] [INSPIRE].

[56] D.M. Pierce, J.A. Bagger, K.T. Matchev and R.-J. Zhang, Precision corrections in the minimal supersymmetric standard model, Nucl. Phys. B 491 (1997) 3 [hep-ph/9606211] [INSPIRE].

[57] L.E. Ibáñez and G.G. Ross, $\mathrm{SU}(2)_{L} \times \mathrm{U}(1)$ symmetry breaking as a radiative effect of supersymmetry breaking in GUTs, Phys. Lett. B 110 (1982) 215 [INSPIRE].

[58] K. Inoue, A. Kakuto, H. Komatsu and S. Takeshita, Aspects of grand unified models with softly broken supersymmetry, Prog. Theor. Phys. 68 (1982) 927 [Erratum ibid. 70 (1983) 330] [INSPIRE].

[59] L.E. Ibáñez, Locally supersymmetric SU(5) grand unification, Phys. Lett. B 118 (1982) 73 [INSPIRE].

[60] J.R. Ellis, D.V. Nanopoulos and K. Tamvakis, Grand unification in simple supergravity, Phys. Lett. B 121 (1983) 123 [INSPIRE].

[61] L. Álvarez-Gaumé, J. Polchinski and M.B. Wise, Minimal low-energy supergravity, Nucl. Phys. B 221 (1983) 495 [INSPIRE].

[62] Particle Data Group collaboration, J. Beringer et al., Review of particle physics (RPP), Phys. Rev. D 86 (2012) 010001 [inSPIRE].

[63] Tevatron Electroweak Working Group, CDF and D0 collaborations, Combination of $C D F$ and D0 results on the mass of the top quark, arXiv:0903.2503 [INSPIRE].

[64] I. Gogoladze, R. Khalid, S. Raza and Q. Shafi, Higgs and sparticle spectroscopy with gauge-Yukawa unification, JHEP 06 (2011) 117 [arXiv:1102.0013] [INSPIRE].

[65] G. Bélanger, F. Boudjema, A. Pukhov and R.K. Singh, Constraining the MSSM with universal gaugino masses and implication for searches at the LHC, JHEP 11 (2009) 026 [arXiv: 0906.5048] [INSPIRE].

[66] H. Baer, S. Kraml, S. Sekmen and H. Summy, Dark matter allowed scenarios for Yukawa-unified SO(10) SUSY GUTs, JHEP 03 (2008) 056 [arXiv:0801.1831] [INSPIRE].

[67] H. Baer, M. Brhlik, C.-H. Chen and X. Tata, Signals for the minimal gauge mediated supersymmetry breaking model at the Fermilab Tevatron collider, Phys. Rev. D 55 (1997) 4463 [hep-ph/9610358] [INSPIRE].

[68] H. Baer, M. Brhlik, D. Castano and X. Tata, $b \rightarrow s \gamma$ constraints on the minimal supergravity model with large $\tan \beta$, Phys. Rev. D 58 (1998) 015007 [hep-ph/9712305] [INSPIRE].

[69] K.S. Babu and C.F. Kolda, Higgs mediated $B^{0} \rightarrow \mu^{+} \mu^{-}$in minimal supersymmetry, Phys. Rev. Lett. 84 (2000) 228 [hep-ph/9909476] [INSPIRE].

[70] A. Dedes, H.K. Dreiner and U. Nierste, Correlation of $B_{s} \rightarrow \mu^{+} \mu^{-}$and $(g-2)_{\mu}$ in minimal supergravity, Phys. Rev. Lett. 87 (2001) 251804 [hep-ph/0108037] [INSPIRE]. 
[71] J.K. Mizukoshi, X. Tata and Y. Wang, Higgs mediated leptonic decays of $B_{s}$ and $B_{d}$ mesons as probes of supersymmetry, Phys. Rev. D 66 (2002) 115003 [hep-ph/0208078] [INSPIRE].

[72] D. Eriksson, F. Mahmoudi and O. Stal, Charged Higgs bosons in minimal supersymmetry: updated constraints and experimental prospects, JHEP 11 (2008) 035 [arXiv:0808.3551] [INSPIRE].

[73] LHCb collaboration, First evidence for the decay $B_{s}^{0} \rightarrow \mu^{+} \mu^{-}$, Phys. Rev. Lett. 110 (2013) 021801 [arXiv: 1211.2674] [INSPIRE].

[74] Heavy Flavor Averaging Group collaboration, Y. Amhis et al., Averages of b-hadron, c-hadron and $\tau$-lepton properties as of early 2012, arXiv:1207.1158 [INSPIRE].

[75] Heavy Flavor Averaging Group collaboration, D. Asner et al., Averages of b-hadron, c-hadron and $\tau$-lepton properties, arXiv:1010.1589 [INSPIRE].

[76] ATLAS collaboration, Observation of a new particle in the search for the standard model Higgs boson with the ATLAS detector at the LHC, Phys. Lett. B 716 (2012) 1 [arXiv:1207.7214] [INSPIRE].

[77] CMS collaboration, Observation of a new boson at a mass of $125 \mathrm{GeV}$ with the CMS experiment at the LHC, Phys. Lett. B 716 (2012) 30 [arXiv:1207.7235] [InSPIRE].

[78] CMS collaboration, Observation of a new boson with mass near $125 \mathrm{GeV}$ in pp collisions at $\sqrt{s}=7$ and $8 \mathrm{TeV}$, JHEP 06 (2013) 081 [arXiv: 1303.4571] [INSPIRE].

[79] WMAP collaboration, G. Hinshaw et al., Nine-year Wilkinson Microwave Anisotropy Probe (WMAP) observations: cosmological parameter results, Astrophys. J. Suppl. 208 (2013) 19 [arXiv:1212.5226] [inSPIRE].

[80] H. Baer et al., Post-LHC7 fine-tuning in the mSUGRA/CMSSM model with a $125 \mathrm{GeV}$ Higgs boson, Phys. Rev. D 87 (2013) 035017 [arXiv:1210.3019] [InSPIRE].

[81] J.R. Ellis, K. Enqvist, D.V. Nanopoulos and F. Zwirner, Observables in low-energy superstring models, Mod. Phys. Lett. A 1 (1986) 57 [InSPIRE].

[82] R. Barbieri and G.F. Giudice, Upper bounds on supersymmetric particle masses, Nucl. Phys. B 306 (1988) 63 [INSPIRE].

[83] H. Baer, V. Barger, P. Huang, A. Mustafayev and X. Tata, Radiative natural SUSY with a 125 GeV Higgs boson, Phys. Rev. Lett. 109 (2012) 161802 [arXiv:1207.3343] [INSPIRE].

[84] I. Gogoladze, F. Nasir and Q. Shafi, Non-universal gaugino masses and natural supersymmetry, Int. J. Mod. Phys. A 28 (2013) 1350046 [arXiv:1212.2593] [InSPIRE].

[85] I. Gogoladze, F. Nasir and Q. Shafi, $\mathrm{SO}(10)$ as a framework for natural supersymmetry, JHEP 11 (2013) 173 [arXiv:1306.5699] [INSPIRE].

[86] CMS collaboration, CMS at the high-energy frontier. Contribution to the update of the european strategy for particle physics, CMS-NOTE-2012-006, CERN, Geneva Switzerland (2012).

[87] H. Baer, M. Brhlik and D. Castano, Constraints on the minimal supergravity model from nonstandard vacua, Phys. Rev. D 54 (1996) 6944 [hep-ph/9607465] [INSPIRE].

[88] J.E. Camargo-Molina, B. Garbrecht, B. O'Leary, W. Porod and F. Staub, Constraining the natural MSSM through tunneling to color-breaking vacua at zero and non-zero temperature, arXiv:1405.7376 [INSPIRE]. 
[89] J.E. Camargo-Molina, B. O'Leary, W. Porod and F. Staub, Stability of the CMSSM against sfermion VEVs, JHEP 12 (2013) 103 [arXiv:1309.7212] [INSPIRE].

[90] I. Gogoladze, R. Khalid, S. Raza and Q. Shafi, $t-b-\tau$ Yukawa unification for $\mu<0$ with a sub-TeV sparticle spectrum, JHEP 12 (2010) 055 [arXiv: 1008.2765] [INSPIRE].

[91] I. Gogoladze, R. Khalid, S. Raza and Q. Shafi, Higgs and sparticle spectroscopy with gauge-Yukawa unification, JHEP 06 (2011) 117 [arXiv:1102.0013] [INSPIRE].

[92] I. Gogoladze, Q. Shafi and C.S. Ün, SO(10) Yukawa unification with $\mu<0$, Phys. Lett. B 704 (2011) 201 [arXiv:1107.1228] [INSPIRE].

[93] I. Gogoladze, Q. Shafi and C.S. Ün, $125 \mathrm{GeV}$ Higgs boson from t-b- $\tau$ Yukawa unification, JHEP 07 (2012) 055 [arXiv: 1203.6082] [INSPIRE].

[94] B. Ananthanarayan, G. Lazarides and Q. Shafi, Top mass prediction from supersymmetric GUTs, Phys. Rev. D 44 (1991) 1613 [inSPIRE].

[95] S.I. Gogolev et al., Total cross-section of the reaction $\pi^{+} d \rightarrow p p$ at pion energies 26-40 MeV, Phys. Lett. B 300 (1993) 24 [INSPIRE].

[96] Q. Shafi and B. Ananthanarayan, Will LEP-2 narrowly miss the Weinberg-Salam Higgs boson?, in Trieste HEP Cosmol., Trieste Italy (1991), pg. 233 [INSPIRE].

[97] I. Gogoladze, Q. Shafi and C.S. Ün, Higgs boson mass from t-b- $\tau$ Yukawa unification, JHEP 08 (2012) 028 [arXiv: 1112.2206] [INSPIRE].

[98] M. Adeel Ajaib, I. Gogoladze, Q. Shafi and C.S. Ün, A predictive Yukawa unified SO(10) model: Higgs and sparticle masses, JHEP 07 (2013) 139 [arXiv:1303.6964] [INSPIRE].

[99] M.A. Ajaib, I. Gogoladze, Q. Shafi and C.S. Ün, Split sfermion families, Yukawa unification and muon $g-2$, JHEP 05 (2014) 079 [arXiv: 1402.4918] [INSPIRE].

[100] H. Baer, S. Raza and Q. Shafi, A heavier gluino from t-b- $\tau$ Yukawa-unified SUSY, Phys. Lett. B 712 (2012) 250 [arXiv:1201.5668] [INSPIRE].

[101] R.D. Peccei and H.R. Quinn, CP conservation in the presence of instantons, Phys. Rev. Lett. 38 (1977) 1440 [INSPIRE].

[102] R.D. Peccei and H.R. Quinn, Constraints imposed by CP conservation in the presence of instantons, Phys. Rev. D 16 (1977) 1791 [InSPIRE].

[103] S. Weinberg, A new light boson?, Phys. Rev. Lett. 40 (1978) 223 [INSPIRE].

[104] F. Wilczek, Problem of strong $p$ and $t$ invariance in the presence of instantons, Phys. Rev. Lett. 40 (1978) 279 [INSPIRE].

[105] H.P. Nilles and S. Raby, Supersymmetry and the strong CP problem, Nucl. Phys. B 198 (1982) 102 [INSPIRE].

[106] J.E. Kim, A common scale for the invisible axion, local SUSY GUTs and saxino decay, Phys. Lett. B 136 (1984) 378 [INSPIRE].

[107] J.E. Kim and H.P. Nilles, The $\mu$ problem and the strong CP problem, Phys. Lett. B 138 (1984) 150 [INSPIRE].

[108] F.D. Steffen, Dark matter candidates - axions, neutralinos, gravitinos and axinos, Eur. Phys. J. C 59 (2009) 557 [arXiv:0811.3347] [InSPIRE]. 
[109] H. Baer and H. Summy, SO(10) SUSY GUTs, the gravitino problem, non-thermal leptogenesis and axino dark matter, Phys. Lett. B 666 (2008) 5 [arXiv:0803.0510] [INSPIRE].

[110] H. Baer, M. Haider, S. Kraml, S. Sekmen and H. Summy, Cosmological consequences of Yukawa-unified SUSY with mixed axion/axino cold and warm dark matter, JCAP 02 (2009) 002 [arXiv:0812.2693] [INSPIRE].

[111] L. Covi, J.E. Kim and L. Roszkowski, Axinos as cold dark matter, Phys. Rev. Lett. 82 (1999) 4180 [hep-ph/9905212] [INSPIRE].

[112] L. Covi, H.-B. Kim, J.E. Kim and L. Roszkowski, Axinos as dark matter, JHEP 05 (2001) 033 [hep-ph/0101009] [INSPIRE].

[113] K.-Y. Choi, J.E. Kim and L. Roszkowski, Review of axino dark matter, J. Korean Phys. Soc. 63 (2013) 1685 [arXiv:1307.3330] [INSPIRE].

[114] H. Baer, S. Kraml, A. Lessa and S. Sekmen, Reconciling thermal leptogenesis with the gravitino problem in SUSY models with mixed axion/axino dark matter, JCAP 11 (2010) 040 [arXiv: 1009.2959] [INSPIRE].

[115] M. Dine, W. Fischler and M. Srednicki, A simple solution to the strong CP problem with a harmless axion, Phys. Lett. B 104 (1981) 199 [INSPIRE].

[116] A.R. Zhitnitsky, On possible suppression of the axion hadron interactions (in Russian), Sov. J. Nucl. Phys. 31 (1980) 260 [Yad. Fiz. 31 (1980) 497] [InSPIRE].

[117] G. Lazarides, C. Panagiotakopoulos and Q. Shafi, Relaxing the cosmological bound on axions, Phys. Lett. B 192 (1987) 323 [InSPIRE].

[118] G. Lazarides, R.K. Schaefer, D. Seckel and Q. Shafi, Dilution of cosmological axions by entropy production, Nucl. Phys. B 346 (1990) 193 [INSPIRE].

[119] J. McDonald, WIMP densities in decaying particle dominated cosmology, Phys. Rev. D 43 (1991) 1063 [INSPIRE].

[120] C. Pallis, Massive particle decay and cold dark matter abundance, Astropart. Phys. 21 (2004) 689 [hep-ph/0402033] [INSPIRE].

[121] K.-Y. Choi, J.E. Kim, H.M. Lee and O. Seto, Neutralino dark matter from heavy axino decay, Phys. Rev. D 77 (2008) 123501 [arXiv:0801.0491] [INSPIRE].

[122] H. Baer, S. Kraml, A. Lessa and S. Sekmen, Thermal leptogenesis and the gravitino problem in the Asaka-Yanagida axion/axino dark matter scenario, JCAP 04 (2011) 039 [arXiv: 1012.3769] [INSPIRE].

[123] H. Baer and A. Lessa, Some necessary conditions for allowing the PQ scale as high as $M_{\mathrm{GUT}}$ in SUSY models with an axino or neutralino LSP, JHEP 06 (2011) 027 [arXiv: 1104.4807] [INSPIRE].

[124] H. Baer, A. Lessa, S. Rajagopalan and W. Sreethawong, Mixed axion/neutralino cold dark matter in supersymmetric models, JCAP 06 (2011) 031 [arXiv:1103.5413] [INSPIRE].

[125] H. Baer, A. Lessa and W. Sreethawong, Coupled Boltzmann calculation of mixed axion/neutralino cold dark matter production in the early universe, JCAP 01 (2012) 036 [arXiv:1110.2491] [INSPIRE].

[126] T. Moroi and L. Randall, Wino cold dark matter from anomaly mediated SUSY breaking, Nucl. Phys. B 570 (2000) 455 [hep-ph/9906527] [INSPIRE]. 
[127] G.B. Gelmini and P. Gondolo, Neutralino with the right cold dark matter abundance in (almost) any supersymmetric model, Phys. Rev. D 74 (2006) 023510 [hep-ph/0602230] [INSPIRE].

[128] G. Gelmini, P. Gondolo, A. Soldatenko and C.E. Yaguna, The effect of a late decaying scalar on the neutralino relic density, Phys. Rev. D 74 (2006) 083514 [hep-ph/0605016] [INSPIRE].

[129] G.B. Gelmini, P. Gondolo, A. Soldatenko and C.E. Yaguna, Direct detection of neutralino dark mattter in non-standard cosmologies, Phys. Rev. D 76 (2007) 015010 [hep-ph/0610379] [INSPIRE].

[130] B.S. Acharya, K. Bobkov, G.L. Kane, P. Kumar and J. Shao, Explaining the electroweak scale and stabilizing moduli in M-theory, Phys. Rev. D 76 (2007) 126010 [hep-th/0701034] [INSPIRE].

[131] B.S. Acharya, K. Bobkov, G.L. Kane, J. Shao and P. Kumar, The $G_{2}$-MSSM: an M-theory motivated model of particle physics, Phys. Rev. D 78 (2008) 065038 [arXiv:0801.0478] [INSPIRE].

[132] B.S. Acharya et al., Non-thermal dark matter and the moduli problem in string frameworks, JHEP 06 (2008) 064 [arXiv:0804.0863] [INSPIRE].

[133] K.J. Bae, H. Baer and A. Lessa, Dark radiation constraints on mixed axion/neutralino dark matter, JCAP 04 (2013) 041 [arXiv: 1301.7428] [INSPIRE].

[134] A. Hryczuk, I. Cholis, R. Iengo, M. Tavakoli and P. Ullio, Indirect detection analysis: wino dark matter case study, JCAP 07 (2014) 031 [arXiv:1401.6212] [INSPIRE].

[135] XENON100 collaboration, E. Aprile et al., Dark matter results from 225 live days of XENON100 data, Phys. Rev. Lett. 109 (2012) 181301 [arXiv:1207.5988] [INSPIRE].

[136] S.P. Martin, A supersymmetry primer, Adv. Ser. Direct. High Energy Phys. 21 (2010) 1 [hep-ph/9709356] [INSPIRE].

[137] L.F. Abbott and P. Sikivie, A cosmological bound on the invisible axion, Phys. Lett. B 120 (1983) 133 [INSPIRE].

[138] J. Preskill, M.B. Wise and F. Wilczek, Cosmology of the invisible axion, Phys. Lett. B 120 (1983) 127 [INSPIRE].

[139] M. Dine and W. Fischler, The not so harmless axion, Phys. Lett. B 120 (1983) 137 [INSPIRE].

[140] M.S. Turner, Cosmic and local mass density of invisible axions, Phys. Rev. D 33 (1986) 889 [INSPIRE].

[141] L. Visinelli and P. Gondolo, Dark matter axions revisited, Phys. Rev. D 80 (2009) 035024 [arXiv:0903.4377] [INSPIRE].

[142] H. Baer et al., Radiative natural supersymmetry: reconciling electroweak fine-tuning and the Higgs boson mass, Phys. Rev. D 87 (2013) 115028 [arXiv:1212.2655] [INSPIRE].

[143] H. Baer, V. Barger and D. Mickelson, Direct and indirect detection of higgsino-like WIMPs: concluding the story of electroweak naturalness, Phys. Lett. B 726 (2013) 330 [arXiv: 1303.3816] [INSPIRE].

[144] CDMS-II collaboration, P.L. Brink et al., Beyond the CDMS-II dark matter search: superCDMS, eConf C 041213 (2004) 2529 [astro-ph/0503583] [INSPIRE]. 
[145] XENON1T collaboration, E. Aprile, The XENON1T dark matter search experiment, Springer Proc. Phys. 148 (2013) 93 [arXiv:1206.6288] [InSPIRE].

[146] A. Bottino, F. Donato, N. Fornengo and S. Scopel, Probing the supersymmetric parameter space by WIMP direct detection, Phys. Rev. D 63 (2001) 125003 [hep-ph/0010203] [INSPIRE].

[147] ICECuBE collaboration, R. Abbasi et al., Limits on a muon flux from neutralino annihilations in the sun with the IceCube 22-string detector, Phys. Rev. Lett. 102 (2009) 201302 [arXiv: 0902 . 2460] [INSPIRE].

[148] G. Jungman, M. Kamionkowski and K. Griest, Supersymmetric dark matter, Phys. Rept. 267 (1996) 195 [hep-ph/9506380] [INSPIRE].

[149] V. Niro, A. Bottino, N. Fornengo and S. Scopel, Investigating light neutralinos at neutrino telescopes, Phys. Rev. D 80 (2009) 095019 [arXiv: 0909.2348] [INSPIRE].

[150] T. Cohen et al., SUSY simplified models at 14, 33 and $100 \mathrm{TeV}$ proton colliders, JHEP 04 (2014) 117 [arXiv: 1311.6480] [inSPIRE]. 